\title{
A mixed formulation for a modification to Darcy equation based on Picard linearization and numerical solutions to large-scale realistic problems
}

\author{
K. B. Nakshatrala and D. Z. Turner
}

\begin{abstract}
In this paper we consider a modification to Darcy equation by taking into account the dependence of viscosity on the pressure. We present a stabilized mixed formulation for the resulting governing equations. Equal-order interpolation for the velocity and pressure is considered, and shown to be stable (which is not the case under the classical mixed formulation). The proposed mixed formulation is tested using a wide variety of numerical examples. The proposed formulation is also implemented in a parallel setting, and the performance of the formulation for large-scale problems is illustrated using a representative problem. Two practical and technologically important problems, one each on enhanced oil recovery and geological carbon-dioxide sequestration, are solved using the proposed formulation. The numerical examples show that the predictions based on Darcy model are qualitatively and quantitatively different from that of the predictions based on the modified Darcy model, which takes into account the dependence of the viscosity on the pressure. In particular, the numerical example on the geological carbon-dioxide sequestration shows that Darcy model over-predicts the leakage into an abandoned well when compared to that of the modified Darcy model. On the other hand, the modified Darcy model predicts higher pressures and higher pressure gradients near the injection well. These predictions have dire consequences in predicting damage and fracture zones, and designing the seal, whose integrity is crucial to the safety of a geological carbon-dioxide sequestration geosystem.
\end{abstract}

\section{INTRODUCTION}

Darcy equation has been successfully employed to model flow in porous media in wide areas of applications ranging from groundwater hydrology, petroleum engineering to food engineering. Henry Darcy has proposed a simple equation to model the flow of an incompressible fluid in rigid porous media, which is popularly referred to as Darcy equation and in some instances as "Darcy

Key words and phrases. Darcy equation; drag coefficient; pressure dependent viscosity; stabilized mixed formulations; variational multiscale formalism; flow through rigid porous media; enhanced oil recovery; parallel implementation. 
law." Darcy has developed this equation empirically based on his experiments on the flow of water through sand beds [1]. (Also see the English translation of Darcy's work by Patricia Bobeck [2].)

It is important to note that Darcy equation is just an approximation of the balance of linear momentum for the fluid flowing through a rigid porous solid (see the discussion in [3, Section 2.5]). The equation is valid under a number of assumptions, see references [3,4 and also [5, Introduction]. Darcy equation merely predicts the flux, and this prediction is not accurate at high pressures. Moreover, in references [5, 6 it has been advocated that Darcy equation is quite good for low flow rates for a fluid like water but is not the case for high flow rates and for dense fluids like mineral oil.

Several extensions to Darcy equation have been developed by various researchers. Two of the early popular ones are by Forchheimer $[\mathbf{7}]$ and Brinkman [8]. Bowen has outlined various models for flow through porous media allowing multiple fluid components and deformation of the porous solid [3]. Recent extensions of Darcy equation can be found in references $\mathbf{9}$ 11. A hierarchy of models for flow through porous media has been presented in [4] of which the simplest model is Darcy equation. One important point to note, which is central to this paper, is that Darcy equation assumes that the coefficient of viscosity (and hence the drag coefficient) to be independent of the pressure in the fluid.

1.1. Pressure dependent viscosity. Certain fluid models do involve flow equations with non-constant viscosity (e.g., a function of pressure or velocity), which are more realistic in modeling enhanced oil recovery and geological carbon-dioxide sequestration. In both enhanced oil recovery and geological carbon-dioxide sequestration, pressure can vary from $0.1 \mathrm{MPa}$ to $100 \mathrm{MPa}$. There is irrefutable experimental evidence that viscosity of mineral oils is not constant and changes drastically with respect to pressure (for example; Bridgman [12], Andrade [13]). In fact, Barus $\mathbf{1 4}$ suggested the following exponential relationship between viscosity and pressure:

$$
\mu(p)=\mu_{0} \exp [\beta p]
$$

where $\beta$ has units $\mathrm{Pa}^{-1}$.

In geological carbon-dioxide sequestration, supercritical carbon-dioxide is pumped into deep saline aquifers or abandoned oil wells. In references $[\mathbf{1 5}, \mathbf{1 6}$, , transport properties (including viscosity) of supercritical carbon-dioxide are presented for various pressures ranging from $0.1 \mathrm{MPa}$ to $8 \mathrm{GPa}$, and for various temperatures. These experimental studies clearly indicate that the viscosity varies exponentially with respect to pressure even for supercritical carbon-dioxide for various temperatures and for a wide range of pressures (varying from $0.1 \mathrm{MPa}$ to $8 \mathrm{GPa}$ ).

It should be emphasized that petroleum reservoir simulations and geological carbon-dioxide sequestration involve heat transfer, mass transfer and phase changes in addition to flow aspects. 
Specifically, one has take into account the attendant geochemical reactions (e.g., see Lichtner [17]). Several mathematical models, numerical formulations, and numerical simulations have been reported in the literature that have taken into account more than the flow aspects. For example, see references $\mathbf{1 8}$ 20. The mathematical model in this paper does not address such issues but just focuses on the need to take into account the dependence of viscosity on the pressure in enhanced oil recovery and geological carbon-dioxide sequestration simulations. Our study is primarily motivated by the fact that the flow characteristics of a fluid whose viscosity depends on pressure can be significantly different from that of the flow characteristics of a fluid with constant viscosity. Some representative prior works on modeling of fluids by taking into account the dependence of viscosity on pressure are $2 \mathbf{2 1}$. 24. In this paper we consider a modification to Darcy equation that takes into account the dependence of viscosity on pressure. The resulting equations will be in mixed form and nonlinear. The unknowns are the velocity and pressure. We present a new stabilized mixed formulation based on the variational multiscale formalism and fixed-point linearization.

1.2. Stabilized mixed finite element formulations. It is well-known that care should be taken to avoid numerical instabilities when dealing with mixed formulations. As discussed by Franca and Hughes [25], a stable mixed formulation either meets or circumvents the LadyzhenskayaBabuška-Brezzi (LBB) inp-sup stability condition [26, 27]. Stabilized methods typically fall in the later category, and the stability is achieved through addition of stabilization terms. But most of the approaches that aim to satisfy LBB condition achieve the stability by restricting the interpolation functions for the independent variables. For example, P1P0 approach in which the velocity unknowns are placed at nodes, and pressure unknowns are for each element/cell. Two other notable works to satisfy the LBB condition are to use either the Raviart-Thomas spaces 28 or Brezzi-Douglas-Marini spaces [29]. In our paper, we do not take such an approach of placing restrictions on the interpolation functions for the independent variables. Instead, we circumvent the LBB condition by adding stabilization terms in a consistent manner, and allow any combination of interpolation functions for the independent variables (in this case, the velocity vector and the pressure).

It should be emphasized that the LBB stability condition is applicable even to mixed formulations based on the finite volume method. As mentioned earlier, a mixed method has to either satisfy the LBB condition in the case of saddle-type formulation, or circumvent the LBB condition by adding stabilization terms to avoid saddle-type problem. In the context of a mixed formulation for Darcy-type equation, the LBB stability condition places a restriction on the choice of the function spaces for the vector-field variable (i.e., the velocity) and the scalar-field variable (i.e., the pressure). It is interesting to note that staggered grids, which is quite popular in the finite volume literature, is a way to satisfy the LBB condition. 
Some popular approaches for developing stabilized finite element formulations are least-squares 30, 31], Galerkin/least-squares (GLS) 32, 33, finite increment calculus (FIC) 34, 35, streamline upwind Petrov-Galerkin $[\mathbf{3 6}]$. Another popular approach for developing stabilized formulations is the variational multiscale framework [37], which has been employed in this paper. The variational multiscale framework has been successfully employed in many studies to develop stabilized formulations for a wide variety of problems: Darcy equation 38,40$]$, Stokes' equation [41, 42], linearized elasticity [43, 44, incompressible Navier-Stokes 45, 46], Fokker-Planck 47.

A huge volume of literature is available on mixed methods and stabilized formulations, and a thorough discussion on these topics is beyond the scope of this paper. Some representative papers on stabilized formulations are $25,32,36,38,40,48,55]$. Some texts concerning stabilized methods are $\left[\begin{array}{ll}56 & 59]\end{array}\right.$. Some representative works on mixed methods in the context of flow through porous media are $38,40,6067$. It should be noted that none of the aforementioned numerical works considered the fluid model considered in this paper.

1.3. Main contributions of this paper. To the best of our knowledge no other prior studies have systematically studied (in a numerical setting) the modified Darcy equation except for Reference [5]. But this paper presents an alternate and simpler formulation than the one presented in Reference 5. The simplicity arises due to the fact that the proposed formulation is obtained by first linearizing the governing equations and then applying the variational multiscale formalism on the resulting equations. This approach facilitates to extend the proposed formulation to other complicated models (e.g., modified Brinkman by taking into account the pressure dependent viscosity, Brinkman-Forchheimer) in a straightforward manner, which may not be the case with the stabilized mixed formulation proposed in Reference [5]). The main contributions of this paper can be summarized as follows:

(a) We considered a realistic modification to Darcy equation by taking into account the dependence of viscosity on the pressure. This implies that the drag coefficient will depend on the pressure. We presented a new stabilized mixed formulation based on the variational multiscale formalism for the resulting nonlinear equations.

(b) We have shown numerically that equal-order interpolation for the velocity and pressure (which is computationally the most convenient) is stable under the proposed formulation. A wide variety of test problems are performed to illustrate the performance of the proposed formulation.

(c) We have implemented the proposed in a parallel setting.

(d) We have solved two practical and technologically important large-scale problems with relevance to enhanced oil recovery and geological carbon-dioxide sequestration. The numerical results have clearly indicated the importance of considering the role of dependence of viscosity on the pressure in these two application areas. 
1.4. An outline of the paper. The remainder of the paper is organized as follows. In Section 2, we present the modified Darcy equation, and present the proposed stabilized mixed finite element formulation, which is based on the variational multiscale formalism. We also present a numerical solution procedure for solving the resulting nonlinear equations. In Section 3 , we illustrate the performance of the proposed formulation on a wide variety of benchmark problems, which are commonly used in the literature for testing mixed formulations. In the same section we also present the numerical results for two large-scale practical problems, which are solved by implementing the proposed formulation in a parallel setting. Conclusions are drawn in Section 4.

\section{GOVERNING EQUATIONS AND STABILIZED FORMULATION}

Let $\Omega \subset \mathbb{R}^{n d}$ be an open and bounded set, where " $n d$ " denotes the number of spatial dimensions. Let $\partial \Omega:=\bar{\Omega}-\Omega$ be the boundary (where $\bar{\Omega}$ is the set closure of $\Omega$ ), which is assumed to be piecewise smooth. A spatial point in $\bar{\Omega}$ is denoted by $\boldsymbol{x}$. The spatial gradient and divergence operators are, respectively, denoted as " $\operatorname{grad}[\cdot] "$ and " $\operatorname{div}[\cdot] "$. Let $\boldsymbol{v}: \Omega \rightarrow \mathbb{R}^{n d}$ denote the velocity field, and $p: \Omega \rightarrow \mathbb{R}$ denote the pressure field. The boundary is divided into two parts, denoted by $\Gamma^{v}$ and $\Gamma^{p}$, such that $\Gamma^{v} \cap \Gamma^{p}=\emptyset$ and $\Gamma^{v} \cup \Gamma^{p}=\partial \Omega$. $\Gamma^{v}$ is the part of the boundary on which normal component of the velocity is prescribed, and $\Gamma^{p}$ is part of the boundary on which pressure is prescribed.

The modified Darcy equations can be written as

$$
\begin{aligned}
& \alpha(p) \boldsymbol{v}+\operatorname{grad}[p]=\rho(\boldsymbol{x}) \boldsymbol{b}(\boldsymbol{x}) \quad \text { in } \Omega \\
& \operatorname{div}[\boldsymbol{v}]=0 \quad \text { in } \Omega \\
& \boldsymbol{v}(\boldsymbol{x}) \cdot \boldsymbol{n}(\boldsymbol{x})=v_{n}(\boldsymbol{x}) \quad \text { on } \Gamma^{v} \\
& p(\boldsymbol{x})=p_{0}(\boldsymbol{x}) \quad \text { on } \Gamma^{p}
\end{aligned}
$$

where $\alpha(p)$ is the drag coefficient (which has dimensions of $\left[\mathrm{ML}^{-3} \mathrm{~T}^{-1}\right]$ ), $p_{0}(\boldsymbol{x})$ is the prescribed pressure, $v_{n}(\boldsymbol{x})$ is the prescribed normal component of the velocity, $\rho(\boldsymbol{x})$ denotes the density of the fluid, $\boldsymbol{b}(\boldsymbol{x})$ is the specific body force, and $\boldsymbol{n}(\boldsymbol{x})$ is the unit outward normal vector to $\partial \Omega$. The drag function is the ratio between viscosity of the fluid and the permeability. Herein we consider the following two forms for drag function

$$
\begin{array}{r}
\alpha(p)=\alpha_{0}(1+\beta p) \\
\alpha(p)=\alpha_{0} \exp [\beta p]
\end{array}
$$

The first equation can be considered as a two-term Taylor's series approximation of the second equation (which is based on the Barus' formula). It is, in general, not possible to obtain analytical solutions for the system of equations (2), especially for complex geometries. Hence, one may have 
to resort to numerical solutions. The main aim of this paper is to present a stabilized mixed formulation to solve the boundary value problem given by equations $(2 \mathrm{a})-(2 \mathrm{~d})$.

Developing numerical formulations for the above equation falls in the realm of mixed methods [27. As mentioned earlier, it is generally agreed upon that care should be taken in developing numerical formulations to avoid numerical instabilities. For example, under the classical Galerkin formulation (which is sometimes referred to as the classical mixed formulation) equal-order interpolation for velocity and pressure is not stable. A mathematical theory that addresses the stability issues with mixed methods is the Ladyzhenskaya-Babuška-Brezzi (LBB) inf-sup condition. Specialized elements and appropriate function spaces have been developed for mixed problems (like Darcy equation, Stokes equation, incompressible Navier-Stokes) to either satisfy or circumvent the LBB stability condition.

In the next subsection we present a new stabilized mixed formulation that is inherently more stable than the classical mixed formulation and can accommodate a larger variety of combinations of interpolation functions for velocity and pressure including the equal-order interpolation. Although the proposed formulation can accommodate many combinations of interpolations for the velocity and pressure, in Section 3 we illustrate the performance of the proposed formulation using equalorder interpolation as other combinations are not computationally attractive.

2.1. A new stabilized mixed formulation for modified Darcy equation. Let $\boldsymbol{w}(\boldsymbol{x})$ and $q(\boldsymbol{x})$ be test functions corresponding to the velocity and pressure, respectively. Let us define the following function spaces, which will be used in the remainder of this paper:

$$
\begin{aligned}
& \mathcal{V}:=\left\{\boldsymbol{v}(\boldsymbol{x}) \in\left(L_{2}(\Omega)\right)^{n d} \mid \operatorname{div}[\boldsymbol{v}] \in L_{2}(\Omega), \boldsymbol{v}(\boldsymbol{x}) \cdot \boldsymbol{n}(\boldsymbol{x})=v_{n}(\boldsymbol{x}) \text { on } \Gamma^{v}\right\} \\
& \mathcal{W}:=\left\{\boldsymbol{w}(\boldsymbol{x}) \in\left(L_{2}(\Omega)\right)^{n d} \mid \operatorname{div}[\boldsymbol{w}] \in L_{2}(\Omega), \boldsymbol{w}(\boldsymbol{x}) \cdot \boldsymbol{n}(\boldsymbol{x})=0 \text { on } \Gamma^{v}\right\} \\
& \mathcal{Q} \equiv H^{1}(\Omega)
\end{aligned}
$$

In using equation $4 \mathrm{c}$ one assumes that $\Gamma^{p} \neq \emptyset$. If $\Gamma^{p}=\emptyset$ then, for well-posedness, $\mathcal{Q}$ should be taken as follows:

$$
\mathcal{Q}:=\left\{p(\boldsymbol{x}) \in H^{1}(\Omega) \mid \int_{\Omega} p(\boldsymbol{x}) \mathrm{d} \Omega=0\right\}
$$

In addition, for the case of $\Gamma^{p}=\emptyset$, we need to satisfy the following compatibility condition on the prescribed data:

$$
\int_{\partial \Omega} v_{n}(\boldsymbol{x}) \mathrm{d} \Gamma=0
$$


For convenience, let us denote the standard $L_{2}$ inner product defined over spatial domain $K$ as $(\cdot ; \cdot)_{K} \cdot$ That is,

$$
(a ; b)_{K} \equiv \int_{K} a \cdot b \mathrm{~d} K \quad \forall a, b
$$

For simplicity, the subscript $K$ will be dropped if $K=\Omega$.

In References 38,40 a stabilized formulation based on the variational multiscale (VMS) formalism has been proposed and analyzed for the case of Darcy equation (which assumes constant drag coefficient). In this paper we extend the variational multiscale formulation to the modified Darcy equation (which gives rise to nonlinear equations). We shall linearize the governing equations (2a) - 2d using a fixed-point procedure as follows:

$$
\begin{aligned}
& \alpha\left(p^{(i-1)}\right) \boldsymbol{v}^{(i)}+\operatorname{grad}\left[p^{(i)}\right]=\rho(\boldsymbol{x}) \boldsymbol{b}(\boldsymbol{x}) \quad \text { in } \Omega \\
& \operatorname{div}\left[\boldsymbol{v}^{(i)}\right]=0 \quad \text { in } \Omega \\
& \boldsymbol{v}^{(i)}(\boldsymbol{x}) \cdot \boldsymbol{n}(\boldsymbol{x})=v_{n}(\boldsymbol{x}) \quad \text { in } \Gamma^{v} \\
& p^{(i)}(\boldsymbol{x})=p_{0}(\boldsymbol{x}) \quad \text { on } \Gamma^{p}
\end{aligned}
$$

Based on the derivation presented in Reference [40], a stabilized mixed formulation for the above equations based on the VMS formalism can be written as follows: Find $\boldsymbol{v}^{(i)}(\boldsymbol{x}) \in \mathcal{V}$ and $p^{(i)}(\boldsymbol{x}) \in \mathcal{Q}$ such that we have

$$
\mathcal{G}_{\text {stab }}\left(\boldsymbol{w}, q ; \boldsymbol{v}^{(i)}, p^{(i)} ; p^{(i-1)}\right)=\mathcal{L}_{\text {stab }}\left(\boldsymbol{w}, q ; p^{(i-1)}\right) \quad \forall \boldsymbol{w}(\boldsymbol{x}) \in \mathcal{W}, q(\boldsymbol{x}) \in \mathcal{Q}
$$

where the functionals $\mathcal{G}_{\text {stab }}$ and $\mathcal{L}_{\text {stab }}$ are, respectively, defined as follows:

$$
\begin{aligned}
\mathcal{G}_{\text {stab }}(\boldsymbol{w}, q ; \boldsymbol{v}, p ; \tilde{p}) & :=(\boldsymbol{w} ; \alpha(\tilde{p}) \boldsymbol{v})-(\operatorname{div}[\boldsymbol{w}] ; p)-(q ; \operatorname{div}[\boldsymbol{v}]) \\
& -\frac{1}{2}\left(\alpha(\tilde{p}) \boldsymbol{w}+\operatorname{grad}[q] ; \alpha^{-1}(\tilde{p})(\alpha(\tilde{p}) \boldsymbol{v}+\operatorname{grad}[p])\right) \\
\mathcal{L}_{\text {stab }}(\boldsymbol{w}, q ; \tilde{p}) & :=(\boldsymbol{w} ; \rho \boldsymbol{b})-\left(\boldsymbol{w} \cdot \boldsymbol{n} ; p_{0}\right)_{\Gamma^{p}}-\frac{1}{2}\left(\alpha(\tilde{p}) \boldsymbol{w}+\operatorname{grad}[q] ; \alpha^{-1}(\tilde{p}) \rho \boldsymbol{b}\right)
\end{aligned}
$$

The terms in the second line of equation $9 \mathrm{a}$ and the last term in $9 \mathrm{~b}$ are referred to as stabilization terms. The factor $1 / 2$ (in front of these terms) is the stabilization parameter. The last term in equation $(9 \mathrm{~b})$ is the stabilization term due to the body force. The stability of the above formulation can be inferred using the mathematical proof outlined in Reference [38].

The proposed numerical algorithm for solving the original system of equations (2) is given in Algorithm 1, and will be based on the above stabilized formulation. Since the formulation is based on the fixed-point linearization, the rate of convergence of the algorithm (if it converges) will be linear with respect to iteration number. Moreover, when $\alpha$ is a constant (that is, for classical Darcy equation), the proposed formulation will converge in one iteration. In a finite element 
implementation, the norm in the stopping criterion (see line 10 in Algorithm 1) can be taken as the Euclidean norm of the vector containing nodal pressures.

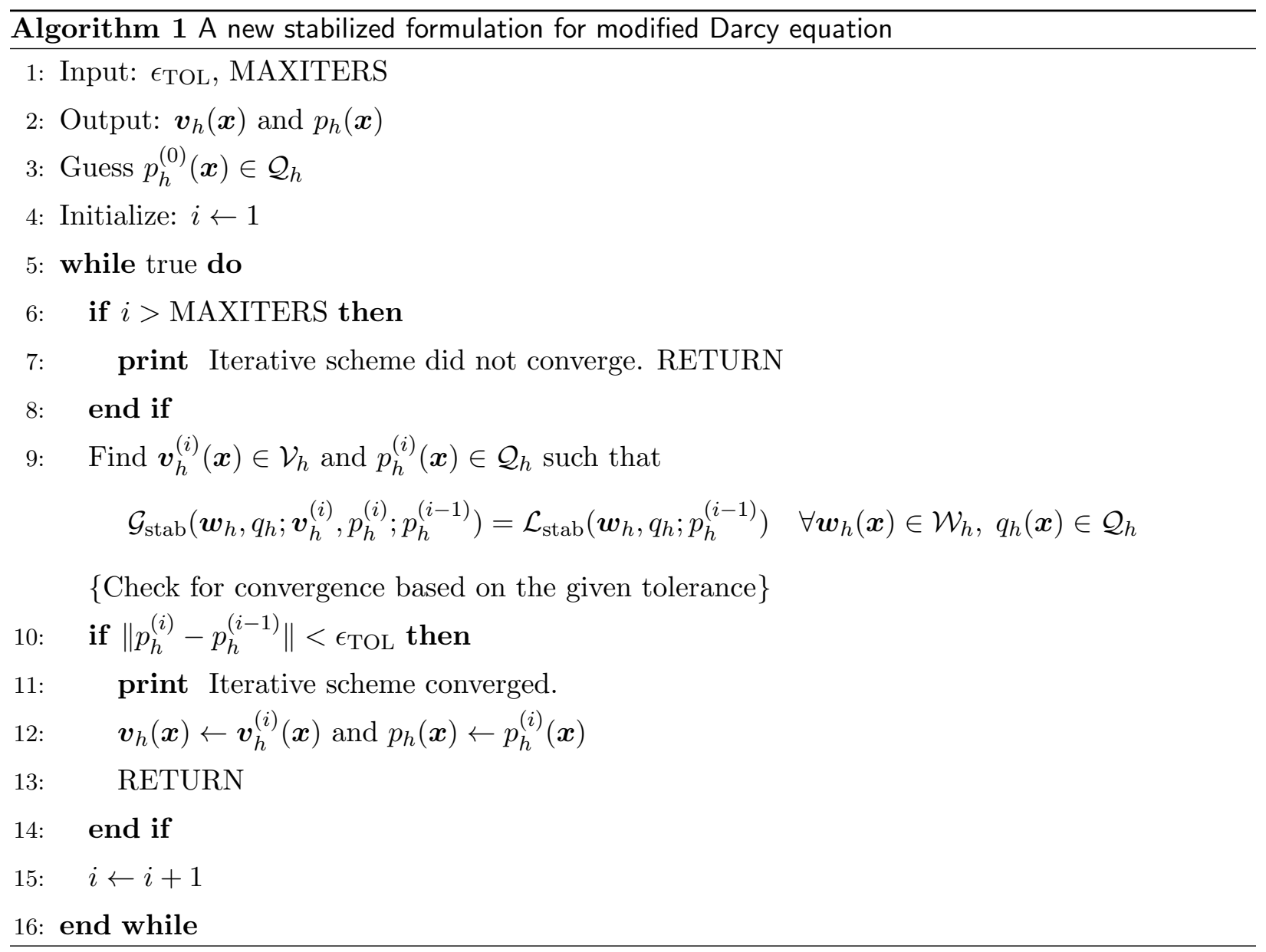

\section{REPRESENTATIVE NUMERICAL RESULTS}

In this section, we present a wide variety of representative numerical results to illustrate the performance of the proposed stabilized formulation. The first few test problems are used to illustrate the accuracy of the proposed formulation on various computational grids as for these test problems analytical solutions can be easily obtained. One test problem is used to illustrate the robustness of the formulation in the case of heterogeneous medium. To the end of the section, the performance of the proposed formulation in a parallel setting is illustrated using a large-scale simulation related to carbon-dioxide sequestration. It should be noted that in all our numerical simulations we have employed low-order Lagrange finite element approximations (i.e., $p=1$ ), and equal-order interpolation for the velocity and pressure.

We shall first non-dimensionalize the governing equations by employing the same non-dimensionalization procedure as given in Reference [5]. We shall present the details for completeness. We define the 
following non-dimensional quantities (which have a superposed bar):

$$
\overline{\boldsymbol{x}}=\frac{\boldsymbol{x}}{L}, \overline{\boldsymbol{v}}=\frac{\boldsymbol{v}}{V}, \bar{p}=\frac{p}{P}, \bar{\alpha}=\frac{\alpha}{\alpha_{\mathrm{ref}}}, \bar{\alpha}_{0}=\frac{\alpha_{0}}{\alpha_{\mathrm{ref}}}, \bar{\rho}=\frac{\rho}{\rho_{\mathrm{ref}}}, \overline{\boldsymbol{b}}=\frac{\boldsymbol{b}}{B}, \bar{\beta}=\beta P, \bar{v}_{n}=\frac{v_{n}}{V}, \bar{p}_{0}=\frac{p_{0}}{P}
$$

where $L, V, P, \alpha_{\text {ref }}, \rho_{\text {ref }}$ and $B$ respectively denote reference length, velocity, pressure, drag coefficient, density and specific body force. The gradient and divergence operators with respect to $\overline{\boldsymbol{x}}$ are denoted as "grad" and "div", respectively. The scaled domain $\Omega_{\text {scaled }}$ is defined as follows: a point in space with position vector $\overline{\boldsymbol{x}} \in \Omega_{\text {scaled }}$ corresponds to the same point with position vector given by $\boldsymbol{x}=\overline{\boldsymbol{x}} L \in \Omega$. Similarly, one can define the scaled boundaries: $\partial \Omega_{\text {scaled }}, \Gamma_{\text {scaled }}^{\mathrm{D}}$, and $\Gamma_{\text {scaled }}^{\mathrm{N}}$. The above non-dimensionalization gives rise to two dimensionless parameters

$$
\mathcal{A}:=\frac{\alpha_{\mathrm{ref}} V L}{P} \text { and } \mathcal{C}:=\frac{\rho_{\mathrm{ref}} L B}{P}
$$

A corresponding non-dimensionalized form of the drag functions given in equation (3) can be written as

$$
\begin{aligned}
& \bar{\alpha}(\bar{p})=\bar{\alpha}_{0}(1+\bar{\beta} \bar{p}) \\
& \bar{\alpha}(\bar{p})=\bar{\alpha}_{0} \exp [\bar{\beta} \bar{p}]
\end{aligned}
$$

We shall write a non-dimensional form of the modified Darcy equation as

$$
\begin{aligned}
& \mathcal{A} \bar{\alpha}(\bar{p}) \overline{\boldsymbol{v}}+\overline{\operatorname{grad}}[\bar{p}]=\mathcal{C} \bar{\rho} \overline{\boldsymbol{b}}(\overline{\boldsymbol{x}}) \quad \text { in } \Omega_{\text {scaled }} \\
& \overline{\operatorname{div}}[\overline{\boldsymbol{v}}]=0 \quad \text { in } \Omega_{\text {scaled }} \\
& \overline{\boldsymbol{v}}(\overline{\boldsymbol{x}}) \cdot \overline{\boldsymbol{n}}(\overline{\boldsymbol{x}})=\bar{v}_{n}(\overline{\boldsymbol{x}}) \quad \text { on } \Gamma_{\text {scaled }}^{\mathrm{N}} \\
& \bar{p}(\overline{\boldsymbol{x}})=\bar{p}_{0}(\overline{\boldsymbol{x}}) \quad \text { on } \Gamma_{\text {scaled }}^{\mathrm{D}}
\end{aligned}
$$

where $\overline{\boldsymbol{n}}(\overline{\boldsymbol{x}})$ is the unit outward normal to the boundary $\partial \Omega_{\text {scaled }}$.

3.1. One-dimensional problem. We shall test the proposed stabilized mixed formulation using a simple one-dimensional problem. Consider the computational domain to be of unit length, and pressures of $\bar{p}_{1}$ and $\bar{p}_{2}$ are respectively prescribed at the left and right ends of the unit domain (see Figure 1). We neglect the body force. The governing equations for this test problem can be written as

$$
\begin{aligned}
& \mathcal{A} \bar{\alpha}(\bar{p}) \bar{v}(\bar{x})+\frac{\mathrm{d} \bar{p}}{\mathrm{~d} \bar{x}}=0 \quad \text { in }(0,1) \\
& \frac{\mathrm{d} \bar{v}}{d \bar{x}}=0 \quad \text { in }(0,1) \\
& \bar{p}(\bar{x}=0)=\bar{p}_{1}, \quad \bar{p}(\bar{x}=1)=\bar{p}_{2}
\end{aligned}
$$


For this test problem, the analytical solutions with the drag functions defined in equation (3) can be written as

$$
\text { for the case } \bar{\alpha}(\bar{p})=\bar{\alpha}_{0}(1+\bar{\beta} \bar{p})\left\{\begin{array}{l}
\bar{p}(\bar{x})=\frac{1}{\bar{\beta}}\left[\left(1+\bar{\beta} \bar{p}_{1}\right)^{1-\bar{x}}\left(1+\bar{\beta} \bar{p}_{2}\right)^{\bar{x}}-1\right] \\
\bar{v}(\bar{x})=\frac{-1}{\mathcal{A} \bar{\alpha}_{0} \bar{\beta}} \ln \left[\frac{1+\bar{\beta} \bar{p}_{2}}{1+\bar{\beta} \bar{p}_{1}}\right]
\end{array}\right.
$$

for the case $\bar{\alpha}(\bar{p})=\bar{\alpha}_{0} \exp [\bar{\beta} \bar{p}]\left\{\begin{array}{l}\bar{p}(\bar{x})=\frac{-1}{\bar{\beta}} \ln \left\{(1-\bar{x}) \exp \left[-\bar{\beta}_{\bar{p}}\right]+\bar{x} \exp \left[-\bar{\beta} \bar{p}_{2}\right]\right\} \\ \bar{v}(\bar{x})=\frac{1}{\mathcal{A} \bar{\alpha}_{0} \beta}\left\{\exp \left[-\bar{\beta} \bar{p}_{2}\right]-\exp \left[-\bar{\beta} \bar{p}_{1}\right]\right\}\end{array}\right.$

In Figures 2 and 3 we compare the numerical solutions against the analytical solutions for various drag functions. In all the cases considered, the proposed numerical formulation performed well. It is important to note that steep gradients in the pressure occur near the boundary for high values of $\beta$ (which is an indicator of the strength of nonlinearity). Figure 4 shows the number of iterations taken by the numerical algorithm with respect to $\beta$. As expected, the number of iterations increases with respect to $\beta$. Figure 4 shows the variation of the residual with respect to the iteration number for various value of $\beta$. The residual decreases monotonically with the iteration number.

3.2. Two-dimensional constant flow problem. The computational domain is a bi-unit square domain, $\bar{\Omega}=[0,1] \times[0,1]$. Barus formula with $\bar{\alpha}_{0}=1$ is employed with two different values of $\bar{\beta}=0.1$ and $\bar{\beta}=0.4$. The body force is neglected. The left and right sides of the domain are prescribed with $\bar{v}_{\bar{x}}=1$. The top and bottom sides of the domain are prescribed with $\bar{v}_{\bar{y}}=0$. The analytical solution is given by

$$
\begin{aligned}
& \bar{v}_{\bar{x}}(\bar{x}, \bar{y})=1, \quad \bar{v}_{\bar{y}}(\bar{x}, \bar{y})=0 \\
& \bar{p}(\bar{x}, \bar{y})=p_{0}-\frac{1}{\bar{\beta}} \ln \left[1-\bar{\alpha}_{0} \bar{\beta}(1-\bar{x}) \exp \left[\bar{\beta} p_{0}\right]\right]
\end{aligned}
$$

We prescribed $p_{0}=1$ at the top-right corner of the computational domain. The finite element meshes used in the numerical simulations are shown in Figure 6. The pressure contours using the proposed stabilized formulation are shown in Figure 7 .

3.3. The quarter five-spot problem. A standard test problem is the quarter five-spot problem. The computational domain is a square in the horizontal plane with injection and production wells at opposite corners along one of the diagonals. The source and sink strengths at injection and production wells are, respectively, taken as $+1 / 4$ and $-1 / 4$. There is no volumetric source/sink (i.e., $\boldsymbol{b}(\boldsymbol{x})=\mathbf{0})$. The five-spot problem is pictorially described in Figure 8 . Figure 9 show the pressure contours using the proposed stabilized formulation. In these simulations we have employed Barus' formula $12 \mathrm{~b}$ with $\bar{\alpha}_{0}=1, \bar{\beta}=0.3, \mathcal{A}=1$, and $\mathcal{B}=0$. The variation of the norm $\left\|\bar{p}_{h}^{(i)}-\bar{p}_{h}^{(i-1)}\right\|$ with respect to iteration number is shown in Figure 10. As one can see from these figures, there are no spurious oscillations in the pressure, and the proposed stabilized formulation performed well. 
Figure 4 shows that the number of iterations increase with an increase in $\beta$, which is a measure of the strength of the nonlinearity. Figure 4 shows that the norm of the residual vector decreases monotonically with respect to the iteration number under the proposed stabilized formulation for the five-spot problem.

3.4. The checkerboard problem. This problem tests the formulation for the case in which there are abrupt changes in the drag coefficient. The geometry and boundary conditions are same as the quarter five-spot test problem. But, for this test problem, the computational domain is divided into four regions as shown in Figure 13 . In regions I and IV, we have taken $\bar{\alpha}_{0}=1$; and in regions II and III, we have taken $\bar{\alpha}_{0}=0.001$. In the numerical simulations we have employed Barus formula with $\bar{\beta}=0.3, \mathcal{A}=1, \mathcal{B}=0$, and have taken $\epsilon_{\mathrm{TOL}}=10^{-9}$. In Figure 14 we have shown the contours of pressure for both four-node quadrilateral and three-node triangular meshes. In Figure 15. we have plotted the variation of the norm $\left\|\bar{p}_{h}^{(i)}-\bar{p}_{h}^{(i-1)}\right\|$ with respect to iteration number. As one can see from these figures, there are no spurious oscillations in the pressure field, and the proposed stabilized formulation performed well.

3.5. Numerical $h$-convergence studies. Consider the computational domain to be $\Omega=$ $(0,1) \times(0,1)$. We have employed Barus formula with $\alpha_{0}=1$ and $\beta=0.5$. The exact solution for the velocity and pressure is given by

$$
v_{x}(x, y)=+\sin (\pi x) \cos (\pi y), \quad v_{y}(x, y)=-\cos (\pi x) \sin (\pi y), \quad p(x, y)=x^{2} y^{2}
$$

The density is taken to be unity, and the specific body force is given by

$b_{x}(x, y)=+\exp \left(x^{2} y^{2} / 2\right) \sin (\pi x) \cos (\pi y)+2 x y^{2}, \quad b_{y}(x, y)=-\exp \left(x^{2} y^{2} / 2\right) \cos (\pi x) \sin (\pi y)+2 x^{2} y$

The normal component of the velocity is prescribed to be zero on the boundary, and $\epsilon_{\mathrm{TOL}}=10^{-9}$. The rate of convergence with respect to mesh refinement is shown in Figure 16. The pressure contours are shown in Figure 17. From these figures it is evident that the proposed algorithm performed well.

3.6. Three-dimensional constant flow. This problem is a three-dimensional version of the patch test, which is employed in Reference 40 to assess the stability of a stabilized formulation for Darcy equation. Herein, we shall use the patch test to assess the stability of proposed stabilized formulation for modified Darcy equation. The formulation is considered to pass the patch test if the flow field matched with the analytical solution up to machine precision.

The computational domain is a cube given by $\Omega=(0,5) \times(0,5) \times(0,5)$. We have employed Barus formula with $\alpha_{0}=1$ and $\beta=0.1$. We have prescribed the normal component of the velocity on $x=0$ and $x=5$ faces to be unity. On the other four outer faces we have prescribed $v_{n}=0$. 
The body force is assumed to be zero, and for uniqueness the pressure at the origin is taken to be zero (that is, $\left.p_{0}(0,0,0)=0\right)$. The analytical solution for the pressure is given by

$$
p(x, y, z)=-\frac{1}{\beta} \ln \left[1+\alpha_{0} \beta x\right]
$$

We have taken $\epsilon_{\mathrm{TOL}}=10^{-9}$ in this numerical simulation. In Figure 18 we have shown the pressure contours obtained using the proposed formulation, and the numerical matched well with the analytical solution.

3.7. Regions with different permeability. This problem considers fully saturated, single phase, single component flow in the region of a production well close to a boundary between regions of permeability that differ by orders of magnitude. Figure ?? shows the problem domain, the production well near the straight interface, and the computational mesh employed in the numerical simulation. The mesh consists of 12,924 eight-node brick linear elements with a total of 63,224 unknowns. The smallest elements (which are near the well) are 0.33 units in diameter while the largest elements (which are near the perimeter of the circle) are 5.0 units in diameter. We have taken $\epsilon_{\mathrm{TOL}}=10^{-12}$. The properties used in this analysis are listed in Table 1. The boundary conditions for this problem consists of no-penetration boundaries at the top and bottom of the domain (except for the opening at the production well). The pressure at the well opening and around the sides is prescribed in a weak fashion with values given in Table 1. The drag function $\bar{\alpha}(\bar{p})$ is calculated using equation $12 \mathrm{a}$ with $\bar{\beta}$ varying between 0.0 and 1.0 .

The exact solution for the velocity streamlines for this problem for constant drag function can be obtained by the method of images and is presented in Reference 68. In region A, the streamlines should curve away from the production well in a radial fashion. In region $\mathrm{B}$, the streamlines should remain straight. Figure 21 shows similar results for the streamlines as produced by the method presented in this work. Note that the streamlines in Region B are straight as predicted by the exact solution. Figure 22 shows a log-log plot of the production rate at the well as a function of $\bar{\beta}$. The production rate was computed as the total flux of fluid $\int \boldsymbol{v}(\boldsymbol{x}) \cdot \boldsymbol{n}(\boldsymbol{x}) \mathrm{d} \Gamma$ across the well opening. Notice that as $\bar{\beta}$ increases, the production rate decreases in a linear fashion. Figure 23 shows the amount of the total production at the well that emanates from region $A$ or $B$. This result is of particular interest in determining the effect of $\bar{\beta}$ on the amount which the well will draw from either region. The results show that although as $\bar{\beta}$ increases the total production decreases, the proportion of the total production that comes from region $A$ or $B$ remains constant.

3.8. $\mathrm{CO}_{2}$ leakage through an abandoned well (large-scale problem). The last numerical example makes an important contribution to the study of geological carbon-dioxide $\left(\mathrm{CO}_{2}\right)$ sequestration into underground aquifers. When $\mathrm{CO}_{2}$ is pumped into an underground aquifer, it can leak through fissures in the surrounding aquitard layers or though man-made penetrations, 
TABLE 1. Parameters used in regions with different permeability problem

\begin{tabular}{ll}
\hline Parameter & Value \\
\hline $\bar{\alpha}_{0}$ region A & 0.001 \\
$\bar{\alpha}_{0}$ region B & 1.0 \\
$\bar{\rho}$ & 1.0 \\
$\overline{\boldsymbol{b}}$ & $(0,0,0)$ \\
$\bar{p}_{0}$ far-field boundary & 1.0 \\
$\bar{p}_{0}$ well opening & $3.333 \times 10^{-4}$ \\
Radius of far-field boundary & 100.0 \\
Radius of well & 1.0 \\
\hline
\end{tabular}

such as abandoned wells. An important question regarding the suitability of certain locations for sequestration involves predicting the leakage rate of $\mathrm{CO}_{2}$ into other aquifers. This numerical example models the leakage of $\mathrm{CO}_{2}$ through an abandoned well as it is injected into an aquifer. The geometry is shown in Figure 24. The diameter of the wells are $0.3 \mathrm{~m}$ and they are located $100 \mathrm{~m}$ apart from each other. The far-field boundary is located at a radius of $500 \mathrm{~m}$. The computational mesh for this problem, which shown in Figure 25, consists of 271,050 eight-node brick linear elements and 284,019 nodes for a total of 1.14 million unknowns. Near the injection well and the abandoned well the element diameter is $0.05 \mathrm{~m}$. Near the far-field boundary the element diameter is $2.5 \mathrm{~m}$. To simplify the problem we assume fully saturated, single component, single phase incompressible flow and that there is no body force, $\overline{\boldsymbol{b}}=(0,0,0)$. The boundary conditions consist of no penetration boundaries on the top and bottom of the aquifers and weakly prescribed pressure boundary conditions along the sides. For aquifer B, a constant reference pressure, $p_{\text {ref }}$, of $2.9315 \times 10^{7} \mathrm{~Pa}$ is prescribed along the outer boundary. For aquifer A, a constant reference pressure of $3.0599 \times 10^{7} \mathrm{~Pa}$ is prescribed. At the injection well, a constant inflow velocity of 0.262 $\mathrm{m} / \mathrm{s}$ is prescribed. The parameters used for this problem are listed in Table $2, \bar{\alpha}(\bar{p})$ is determined according to the exponential function given in equation $(12 \mathrm{~b})$, with $\bar{\beta}$ varying from 0.0 to 1.0 . We have again taken $\epsilon_{\mathrm{TOL}}=10^{-12}$.

This problem was solved in parallel setting with 32 processors, on the Tri-Lab Linux Capacity Cluster, using Aria computer code 69 . Load balancing across the processors was achieved using the Zoltan 70 package. The total CPU time for a single run with a given $\beta$ was 35 minutes with an average memory use on each processor of 400 MB. A complete investigation into the parallel scalability of this algorithm is intended for future work.

Figure 26 shows contours of the pressure for the minimum and maximum values of $\bar{\beta}$. Notice that as $\bar{\beta}$ increases, the pressure in the region surrounding the injection well increases. Figure 27 
shows the magnitude of the velocity for the maximum and minimum values of $\bar{\beta}$. As $\bar{\beta}$ increases, the velocity magnitude in the region of the abandoned well decreases suggesting that not including the pressure dependent viscosity under predicts the leakage rate. Figure 28 shows the ratio of injection rate to leakage rate as $\beta$ increases. The injection rate was computed as the total flux of fluid $\int \boldsymbol{v}(\boldsymbol{x}) \cdot \boldsymbol{n}(\boldsymbol{x}) \mathrm{d} \Gamma$ across the injection well opening. Likewise, the leakage rate was computed as the total flux of fluid across a cross-section of the abandoned well at the bottom of the aquifer B. In References $[\mathbf{7 1}, \mathbf{7 2}$, the authors present transient results for a similar simulation for multiphase flow of $\mathrm{CO}_{2}$ and brine, with different saturations, and constant viscosity. They observe peak ratios of leakage rate to injection rate in the range of $0.2 \%$ to $0.42 \%$. For the simulation presented in this work, the ratio is between $1 \%$ and $10 \%$. Clearly, the assumptions of fully saturated, single phase flow do not accurately capture the leakage rate, but the results are meaningful in that they show, in general, an over-prediction of the leakage rate if a pressure dependent viscosity is not accounted for. As $\bar{\beta}$ increases, the amount $\mathrm{CO}_{2}$ that leaks into aquifer $\mathrm{B}$ is decreased significantly. Such results suggest that a pressure dependent viscosity model has a substantial effect on the predicted leakage rate.

TABLE 2. $\mathrm{CO}_{2}$ leakage through an abandoned well: problem parameters

\begin{tabular}{ll}
\hline Parameter & Value \\
\hline $\bar{\alpha}_{0}$ aquifer A & 1.0 \\
$\bar{\alpha}_{0}$ aquifer B & 1.0 \\
$\bar{\alpha}_{0}$ inside the wells & 100 \\
$\bar{p}_{0}$ aquifer A & 1.0 \\
$\bar{p}_{0}$ aquifer B & 0.95 \\
$\beta$ & $1.0 \times 10^{-7}$ \\
$\boldsymbol{v} \cdot \boldsymbol{n}$ at inflow & 0.262 \\
\hline
\end{tabular}

\section{CONCLUDING REMARKS}

We have considered a modification to Darcy equation by taking into account the dependence of viscosity on the pressure, which has been observed in many experiments on organic liquids. We have developed a new mixed stabilized formulation for the modified Darcy equation. We have also presented a numerical solution procedure to solve the resulting nonlinear equations. It has been shown numerically that equal-order interpolation for the velocity and pressure is stable under the proposed stabilized mixed formulation, which is not the case with the classical mixed formulation. Using representative problems, it has been shown that

(a) the proposed stabilized mixed formulation performs well, and 
(b) the results predicted by the standard Darcy model are qualitatively and quantitatively different from that of the predictions based on the modified Darcy model.

It has been observed that the dependence of viscosity on the pressure drastically alters the pressure profile in the domain, and creates steep gradients near the boundary of the domain. Using a representative problem with relevance to geological carbon-dioxide sequestration, it has been shown that the standard Darcy model over-predicts the velocity of the fluid in the abandon well in comparison with the modified Darcy model. This prediction will serious consequences in designing the ceiling of the cap rock, which is one of the main mechanisms for the safety of a geological carbon-dioxide geosystem. Another important point to be noted is that the modified Darcy model predicts higher

pressures than that of the Darcy model, which will have implications in modeling damage and fracture of the porous solid.

As a part of future work on the numerical front, one can extend the current work to solve problems with much higher $\beta$ values by employing continuation-type methods (that is, to solve the problem for a high $\beta$ using information from the solution at a lower $\beta$ ). Another interesting future numerical work can be designing preconditioners for these kinds of nonlinear problems. A possible future work on the modeling front is to model the damage and fracture of the porous solid along with the flow aspects by taking into account the dependence of the viscosity on the pressure.

\section{ACKNOWLEDGMENTS}

The first author (K. B. Nakshatrala) was supported in part by the Department of Energy through the Subsurface Biogeochemical Research (SBR) Program. The authors also acknowledge the financial support from Sandia National Laboratories through the Laboratory Directed Research and Development program. The opinions expressed in this paper are those of the authors and do not necessarily reflect that of the sponsors. The authors thank Pat Notz and Mario Martinez for their insightful discussions on this topic.

\section{References}

[1] H. Darcy. Les Fontaines Publiques de la Ville de Dijon. Victor Dalmont, Paris, 1856.

[2] P. Bobeck. The Public Fountains of the City of Dijon. Kendall Hunt Publishing, Dubuque, Iowa, USA, 2004.

[3] R. Bowen. Porous Elasticity: Lectures on the Elasticity of Porous Materials as an Application of the Theory of Mixtures. Available online: http://repository.tamu.edu/handle/1969.1/2500, Texas A\&M University, 2010.

[4] K. R. Rajagopal. On a hierarchy of approximate models for flows of incompressible fluids through porous solids. Mathematical Models and Methods in Applied Sciences, 17:215-252, 2007.

[5] K. B. Nakshatrala and K. R. Rajagopal. A numerical study of fluids with pressure dependent viscosity flowing through a rigid porous medium. International Journal for Numerical Methods in Fluids, DOI: 10.1002/fld.2358, 2010 . 
[6] D. Munaf, A. S. Wineman, K. R. Rajagopal, and D. W. Lee. A boundary value problem in groundwater motion analysis-comparisons based on Darcy's law and the continuum theory of mixtures. Mathematical Modeling and Methods in Applied Science, 3:231-248, 1993.

[7] P. Forchheimer. Wasserbewegung durch Boden. Zeitschrift des Vereines Deutscher Ingeneieure, 45:1782-1788, 1901.

[8] H. C. Brinkman. On the permeability of the media consisting of closely packed porous particles. Applied Scientific Research, A1:81-86, 1947.

[9] J. M. D. Medeiros, J. M. Gurgel, and F. Marcondes. Numerical analysis of natural convection in porous media: The influence of non-Darcian terms and thermal dispersion. Journal of Porous Media, 9:235-250, 2006.

[10] K. Al-Farhany and A. Turan. Non-Darcy effects on conjugate double-diffusive natural convection in a variable porous layer sandwiched by finite thickness walls. International Journal of Heat and Mass Transfer, 54:2868$2879,2011$.

[11] T. R. Mahapatra, D. Pal, and S. Mondal. Influence of thermal radiation on non-Darcian natural convection in a square cavity filled with fluid saturated porous medium of uniform porosity. Nonlinear Analysis: Modelling and Control, 17:223-237, 2012.

[12] P. W. Bridgman. The Physics of High Pressure. MacMillan Company, New York, USA, 1931.

[13] E. N. da. C. Andrade. Viscosity of liquids. Proceedings of the Royal Society of London, Series A, 215:36-43, 1952.

[14] C. Barus. Isotherms, isopiestics and isometrics relative to viscosity. American Journal of Science, 45:87-96, 1893.

[15] V. Vesovic, W. A. Wakeham, G. A. Olchowy, J. V. Sengers, J. T. R. Watson, and J. Millat. The transport properties of carbon dioxide. Journal of Physical and Chemical Reference Data, 19:763-808, 1990.

[16] E. H. Abramson. Viscosity of carbon dioxide measured to a pressure of 8 GPa and temperature of 673 K. Physical Review E, 80(021201), 2009.

[17] P. C. Lichtner. Continuum formulation of multicomponent-multiphase reactive transport. Reviews in Mineralogy and Geochemistry, 34:1-81, 1996.

[18] P. C. Lichtner. Continuum model for simultaneous chemical reactions and mass transport in hydrothermal systems. Geochimica et Cosmochimica Acta, 49:779-800, 1985.

[19] C. Lu and P. C. Lichtner. High resolution numerical investigation on the effect of convective instability on long term $\mathrm{CO}_{2}$ storage in saline aquiferes. Journal of Physics: Conference Series, 78:012042, 2007.

[20] F. Marcondes and K. Sepehrnoori. An element-based finite-volume method approach for heterogeneous and anisotropic compositional reservoir simulation. Journal of Petroleum Science and Engineering, 73:99-106, 2010.

[21] J. Málek, J. Nečas, and K. R. Rajagopal. Global analysis of the flows of fluids with pressure-dependent viscosities. Archive for Rational Mechanics and Analysis, 165:243-269, 2002.

[22] S. C. Subramaniam and K. R. Rajagopal. A note on the flow through porous solids at high pressures. Computers and Mathematics with Applications, 53:260-275, 2007.

[23] M. Buliček, J. Málek, and K. R. Rajagopal. Navier's slip and evolutionary Navier-Stokes-like systems with pressure and shear-rate depedent viscosity. Indiana University Mathematics Journal, 56:51-85, 2007.

[24] K. Kannan and K. R. Rajagopal. Flow through porous media due to high pressure gradients. Applied Mathematics and Computation, 199:748-759, 2008.

[25] L. P. Franca and T. J. R. Hughes. Two classes of mixed finite element methods. Computer Methods in Applied Mechanics and Engineering, 69:89-129, 1988. 
[26] I. Babuška. Error bounds for finite element methods. Numerische Mathematik, 16:322-333, 1971.

[27] F. Brezzi and M. Fortin. Mixed and Hybrid Finite Element Methods, volume 15 of Springer series in computational mathematics. Springer-Verlag, New York, USA, 1991.

[28] P. A. Raviart and J. M. Thomas. A mixed finite element method for 2nd order elliptic problems. In Mathematical Aspects of the Finite Element Method, pages 292-315, Springer-Verlag, New York, 1977.

[29] F. Brezzi, J. Douglas, and L. D. Marini. Two families of mixed elements for second order elliptic problems. Numerische Mathematik, 47:217-235, 1985.

[30] P. B. Bochev and M. D. Gunzburger. Least-Squares Finite Element Methods. Springer, New York, USA, 2009.

[31] B. Jiang. The Least-Squares Finite Element Method: Theory and Applications in Computational Fluid Dynamics and Electromagnetics. Springer-Verlag, New York, USA, 1998.

[32] T. J. R. Hughes, L. Franca, and G. Hulbert. A new finite element formulation for computational fluid dynamics: VIII. The Galerkin/least-squares method for advective-diffusive equations. Computer Methods in Applied Mechanics and Engineering, 73:173-189, 1989.

[33] C. Baiocchi, F. Brezzi, and L. Franca. Virtual bubbles and Galerkin-least-squares type methods (Ga.L.S.). Computer Methods in Applied Mechanics and Engineering, 105:125-141, 1993.

[34] E. Onate. A stabilized finite element method for incompressible viscous flows using a finite increment calculus formulation. Computer Methods in Applied Mechanics and Engineering, 182:355-370, 2000.

[35] E. Onate. Possibilities of finite calculus in computational mechanics. International Journal for Numerical Methods in Engineering, 60:255-281, 2004.

[36] A. N. Brooks and T. J. R. Hughes. Streamline-upwind/Petrov-Galerkin methods for convection dominated flows with emphasis on the incompressible Navier-Stokes equations. Computer Methods in Applied Mechanics and Engineering, 32:199-259, 1982.

[37] T. J. R. Hughes. Multiscale phenomena: Green's functions, the Dirichlet-to-Neumann formulation, subgrid scale models, bubbles and the origins of stabilized methods. Computer Methods in Applied Mechanics and Engineering, 127:387-401, 1995.

[38] A. Masud and T. J. R. Hughes. A stabilized mixed finite element method for Darcy flow. Computer Methods in Applied Mechanics and Engineering, 191:4341-4370, 2002.

[39] T. J. R. Hughes, A. Masud, and J. Wan. A stabilized mixed discontinuous Galerkin method for Darcy flow. Computer Methods in Applied Mechanics and Engineering, 195:3347-3381, 2006.

[40] K. B. Nakshatrala, D. Z. Turner, K. D. Hjelmstad, and A. Masud. A stabilized mixed finite element formulation for Darcy flow based on a multiscale decomposition of the solution. Computer Methods in Applied Mechanics and Engineering, 195:4036-4049, 2006.

[41] A. Masud. A stabilized mixed finite element method for Darcy-Stokes flow. International Journal for Numerical Methods in Fluids, 54:665-681, 2007.

[42] D. Z. Turner, K. B. Nakshatrala, and K. D. Hjelmstad. On the stability of bubble functions and a stabilized mixed finite element formulation for the Stokes problem. International Journal for Numerical Methods in Fluids, 60:1291-1314, 2009.

[43] A. Masud and K. Xia. A stabilized mixed finite element method for nearly incompressible elasticity. Journal of Applied Mechanics, 72:711-720, 2005.

[44] K. B. Nakshatrala, A. Masud, and K. D. Hjelmstad. On finite element formulations for nearly incompressible linear elasticity. Computational Mechanics, 41:547-561, 2008. 
[45] A. Masud and R. A. Khurram. A multiscale finite element method for the incompressible Navier-Stokes equations. Computer Methods in Applied Mechanics and Engineering, 195:1750-1777, 2006.

[46] D. Z. Turner, K. B. Nakshatrala, and K. D. Hjelmstad. A variational multiscale Newton-Schur approach for the incompressible Navier-Stokes equations. International Journal for Numerical Methods in Fluids, 62:119-137, 2010.

[47] A. Masud and L. A. Bergman. Application of multiscale finite element methods to the solution of the FokkerPlanck equation. Computer Methods in Applied Mechanics and Engineering, 194:1513-1526, 2005.

[48] I. Babuška, J. T. Oden, and J. K. Lee. Mixed-hybrid finite element approximations of second-order elliptic boundary-value problems. Computer Methods in Applied Mechanics and Engineering, 11:175-206, 1977.

[49] J. T. Oden and O.-P. Jacquotte. Stability of some mixed finite element methods for Stokesian flows. Computer Methods in Applied Mechanics and Engineering, 43:231-247, 1984.

[50] T. J. R. Hughes, L. Franca, and M. Balestra. A new finite element formulation for computational fluid dynamics: V. Circumventing the Babuska-Brezzi condition: A stable Petrov-Galerkin formulation of the Stokes problem accommodating equal-order interpolations. Computer Methods in Applied Mechanics and Engineering, 59:85-99, 1986.

[51] J. Doughlas and J. Wang. An absolutely stabilized finite element method for the Stokes problem. Mathematics of Computation, 52:495-508, 1989.

[52] L. P. Franca, S. L. Frey, and T. J. R. Hughes. Stabilized finite element methods: I. Application to the advectivediffusive model. Computer Methods in Applied Mechanics and Engineering, 95:253-276, 1992.

[53] L. P. Franca and A. Russo. Unlocking with residual-free bubbles. Computer Methods in Applied Mechanics and Engineering, 142:361-364, 1997.

[54] E. Burman and P. Hansbo. Stabilized Crouzeix-Raviart Elements for the Darcy-Stokes Problem. Numerical Methods for Partial Differential Equations, 21:986-997, 2005.

[55] M. R. Correa and A. F. D. Loula. Unconditionally stable mixed finite element methods for Darcy flow. Computer Methods in Applied Mechanics and Engineering, 197:1525-1540, 2008.

[56] A. Quarternio and A. Valli. Numerical Approximation of Partial Differential Equations. Springer series in computational mathematics. Springer-Verlag, New York, USA, 1997.

[57] H.-G. Roos, M. Stynes, and L. Tobiska. Numerical Methods for Singularly Perturbed Differential Equations. Springer-Verlag, New York, USA, 1996.

[58] Z. Chen, G. Huan, and Y. Ma. Computational Methods for Multiphase Flows in Porous Media. Society for Industrial and Applied Mathematics, Philadelphia, USA, 2006.

[59] J. Donea and A. Huerta. Finite Element Methods for Flow Problems. John Wiley \& Sons, Inc., Chichester, UK, 2003.

[60] R. E. Ewing and R. F. Heinemann. Convergence analysis of an approximation of miscible displacement in porous media by mixed finite elements and a modified method of characteristics. Computer Methods in Applied Mechanics and Engineering, 47:73-92, 1984.

[61] G. Chavent, G. Cohen, and J. Jaffre. Discontinuous upwinding and mixed finite element for two-phase flows in reservoir simulation. Computer Methods in Applied Mechanics and Engineering, 47:93-118, 1984.

[62] R. E. Ewing and R. F. Heinemann. Mixed finite element approximation of phase velocities in compositional reservoir simulation. Computer Methods in Applied Mechanics and Engineering, 47:161-175, 1984. 
[63] L. J. Durlofsky. Accuracy of mixed and control volume finite element approximations to Darcy velocity and related quantities. Water Resources Research, 30:965-973, 1994.

[64] T. Arbogast, M. F. Wheeler, and I. Yotov. Mixed finite elements for elliptic problems with tensor coefficients as cell-centered finite differences. SIAM Journal on Numerical Analysis, 34:828-852, 1997.

[65] L. Bergamaschi, S. Mantica, and G. Manzini. A mixed finite element-volume formulation of the black oil model. SIAM Journal of Scientific Computing, 20:970-997, 1998.

[66] F. Brezzi, T. J. R. Hughes, L. D. Marini, and A. Masud. Mixed discontinuous Galerkin method for Darcy flow. SIAM Journal of Scientific Computing, 22:119-145, 2005.

[67] E. Burman and P. Hansbo. A unified stabilized method for Stokes' and Darcy's equations. Journal of Computational and Applied Mathematics, 198:35-51, 2007.

[68] J. Bear. Hydraulics of Groundwater. McGraw-Hill, New York, USA, 1979.

[69] P. Notz, S. R. Subia, M. Hopkins, H. K. Moffat, and D. R. Noble. Aria 1.5 User Manual. Technical report, Sandia Report SAND2007-2734, Sandia National Laboratories, 2007.

[70] K. Devine, E. Boman, R. Heaphy, B. Hendrickson, and C. Vaughan. Zoltan data management services for parallel dynamic applications. Computing in Science and Engineering, 4:90-97, 2002.

[71] A. Ebigbo, H. Class, and R. Helmig. $\mathrm{CO}_{2}$ leakage through an abandoned well: problem-oriented benchmarks. Computers \& Geoscience, 11:103-115, 2007.

[72] J. M. Nordbotten, M. A. Celia, S. Bachu, and H. K. Dahle. Semianalytical solution for $\mathrm{CO}_{2}$ leakage through an abandoned well. Environmental Science and Technology, 39:602-611, 2005. 


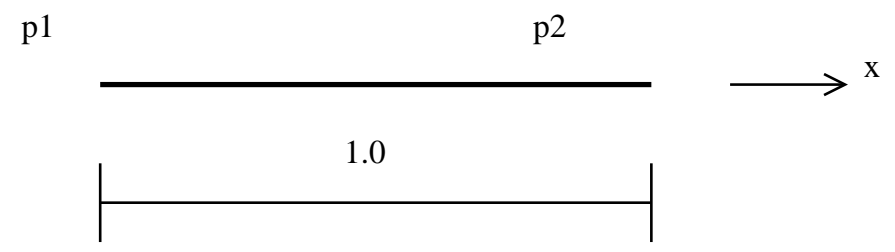

Figure 1. A pictorial description of the one-dimensional problem.
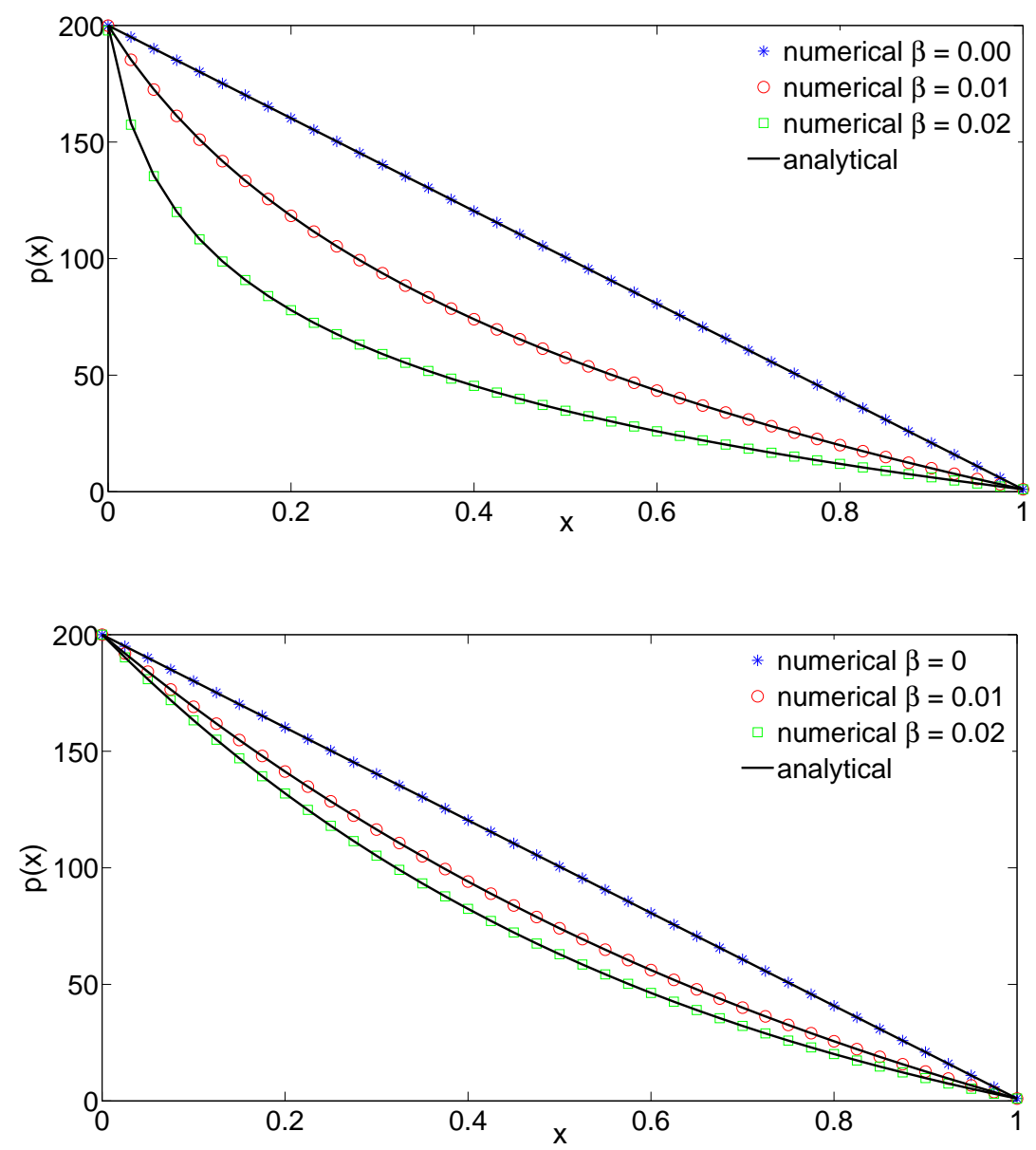

Figure 2. One-dimensional problem: In the top figure Barus formula is employed, and in the bottom figure linear variation of viscosity with respect to pressure is employed. Pressure is plotted along $x$ for various values of $\beta$. In this numerical example we have taken $\bar{\alpha}_{0}=1, \bar{p}(\bar{x}=0)=200, \bar{p}(\bar{x}=1)=1$, and $\epsilon_{\mathrm{TOL}}=10^{-10}$. 


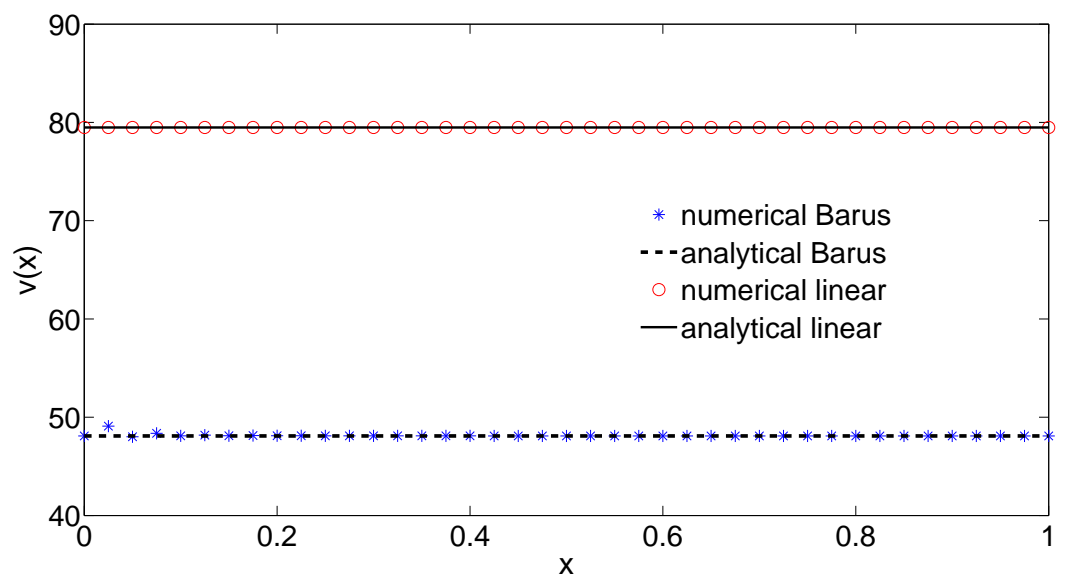

Figure 3. One-dimensional problem: Velocity is plotted along $x$ for various values of $\beta$. In this numerical example we have taken $\bar{\alpha}_{0}=1, \bar{\beta}=0.02, \bar{p}(\bar{x}=0)=200$, $\bar{p}(\bar{x}=1)=1$, and $\epsilon_{\mathrm{TOL}}=10^{-10}$.

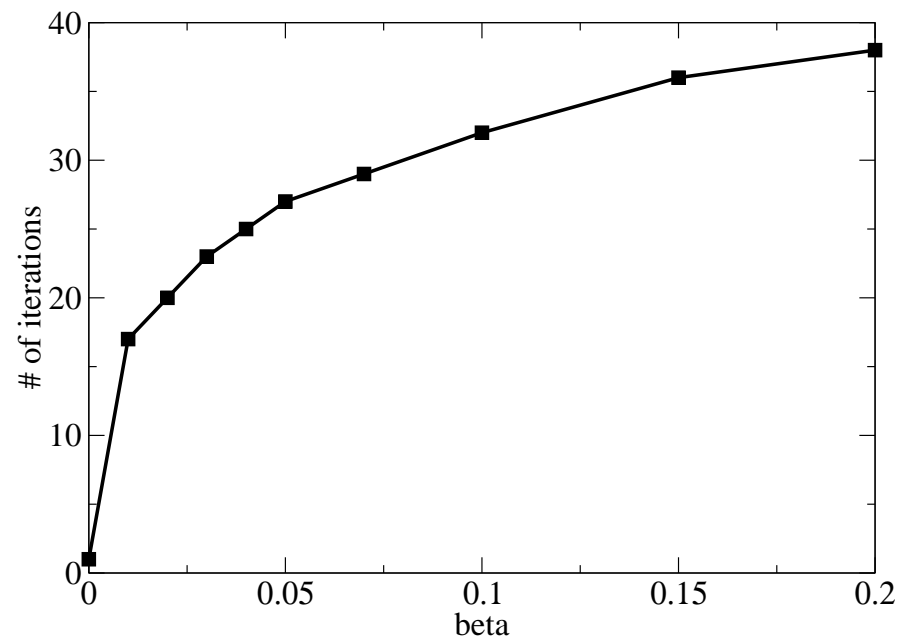

Figure 4. One-dimensional problem: This figure shows the number of iterations taken by the numerical algorithm for various values of $\beta$, which is an indicator of the strength of the nonlinearity. 


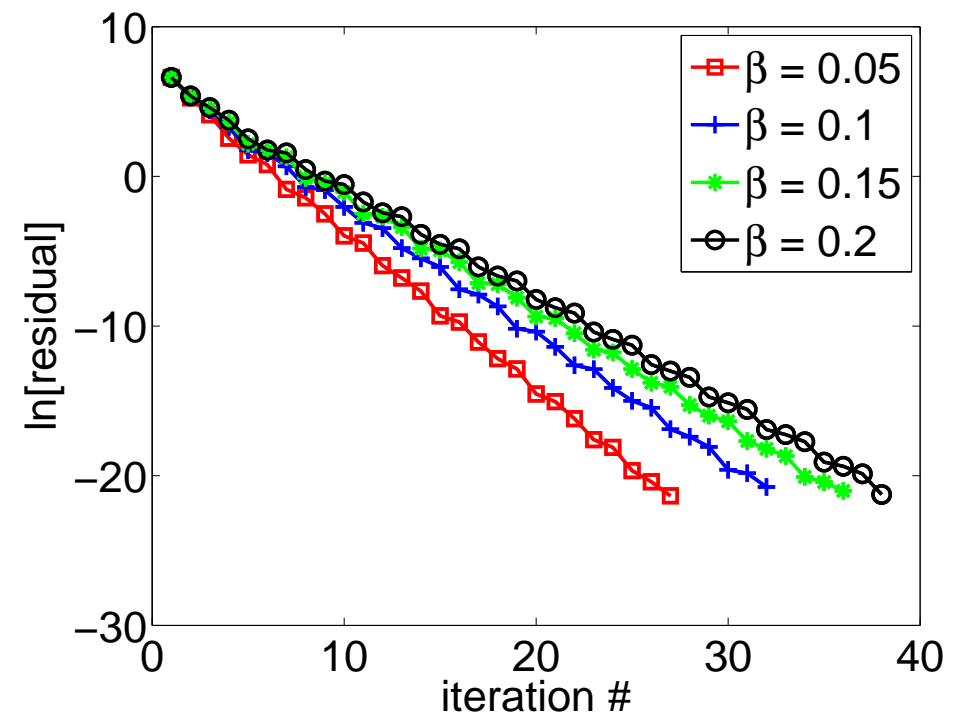

Figure 5. One-dimensional problem: This figure shows the variation of the residual with respect to iteration number for various values of $\beta$. Note that the $y$-axis is natural logarithm of the residual. 

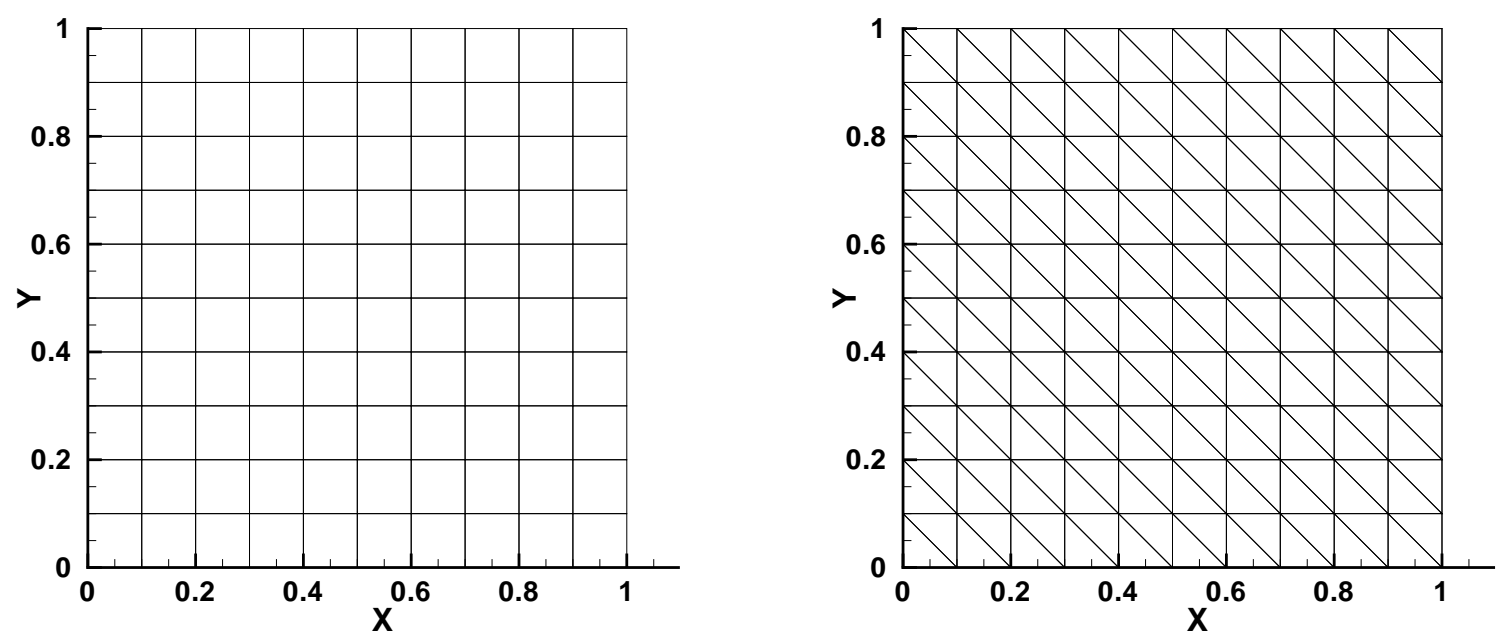

Figure 6. Two-dimensional constant flow problem: Four-node quadrilateral (left) and three-node triangular (right) meshes used in numerical simulations. 

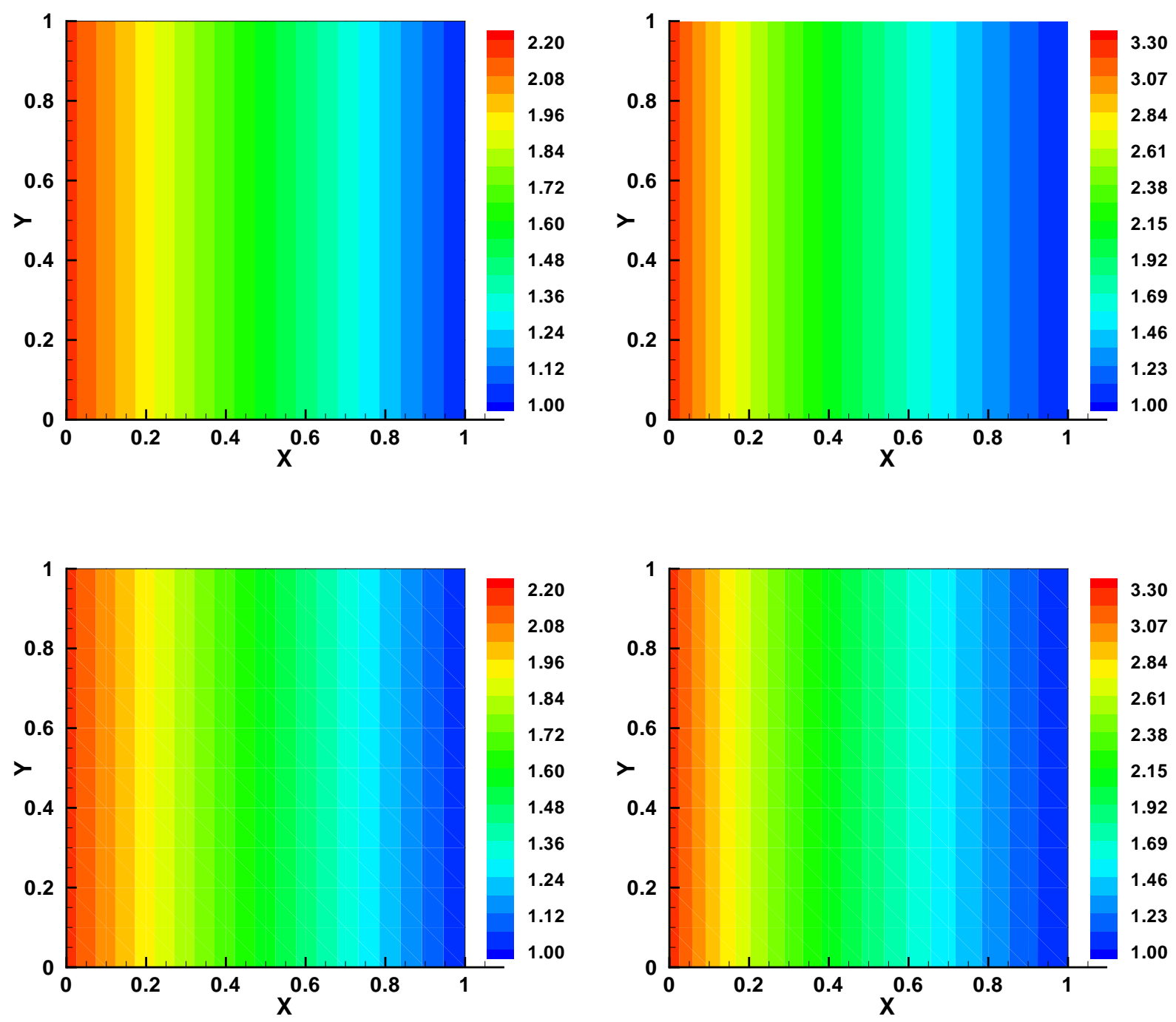

Figure 7. Two-dimensional constant flow problem: Barus formula is employed with $\alpha_{0}=1$, and the tolerance is taken to be $\epsilon_{\mathrm{TOL}}=10^{-10}$. For the left figure we have used $\beta=0.1$ and for the right figure we have used $\beta=0.4$. Four-node quadrilateral mesh is used for top figures, and three-node triangular mesh is used for bottom figures. The numerical results matched well with the analytical solution. 


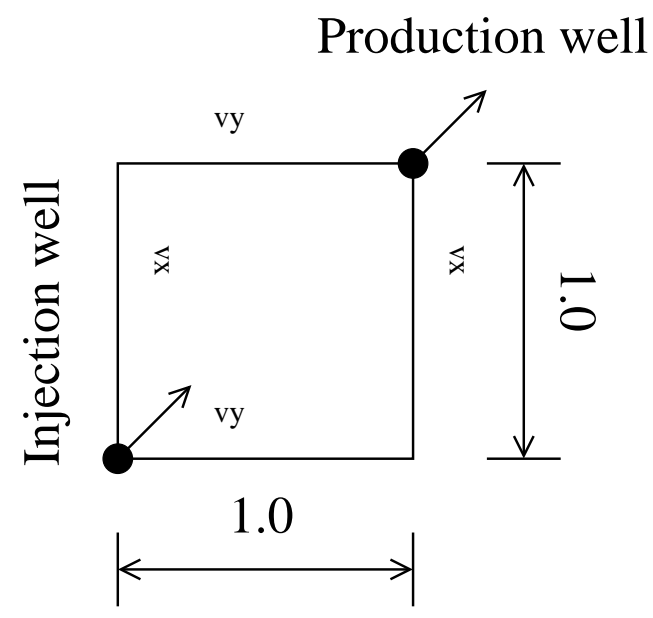

Figure 8. A pictorial description of the quarter five-spot problem. 


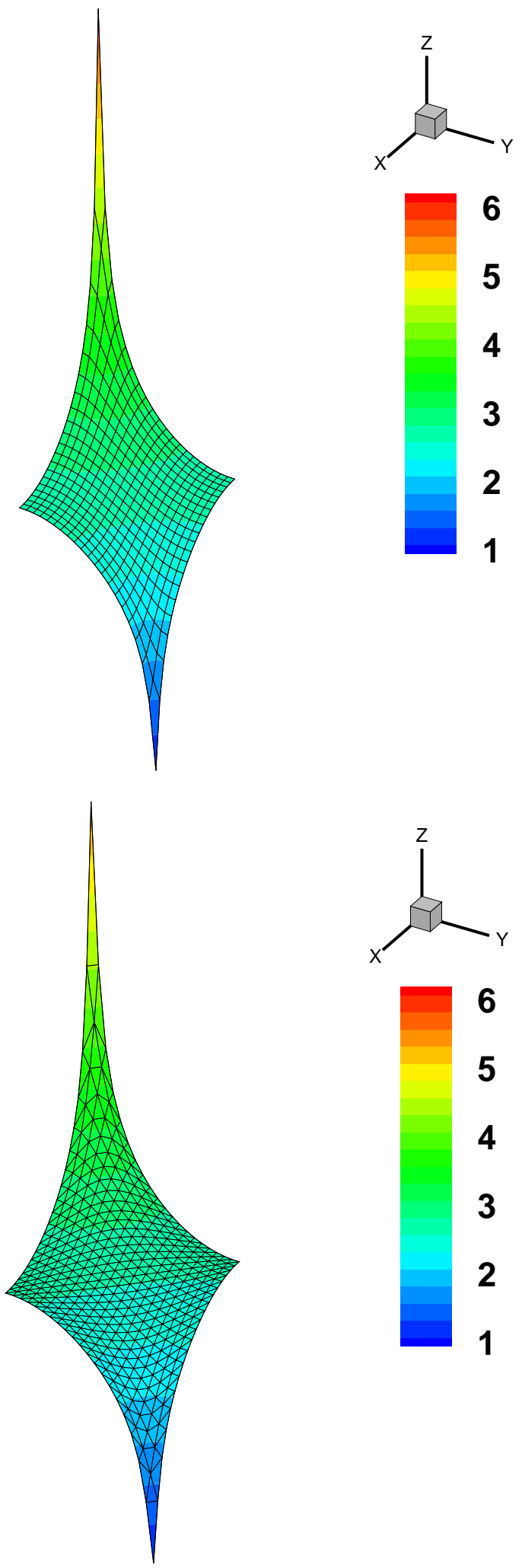

Figure 9. Five-spot problem: Pressure contours using four-node quadrilateral (top) and three-node triangular (bottom) elements. We have used 21 nodes along each side of the computational domain. ${ }^{26}$ 


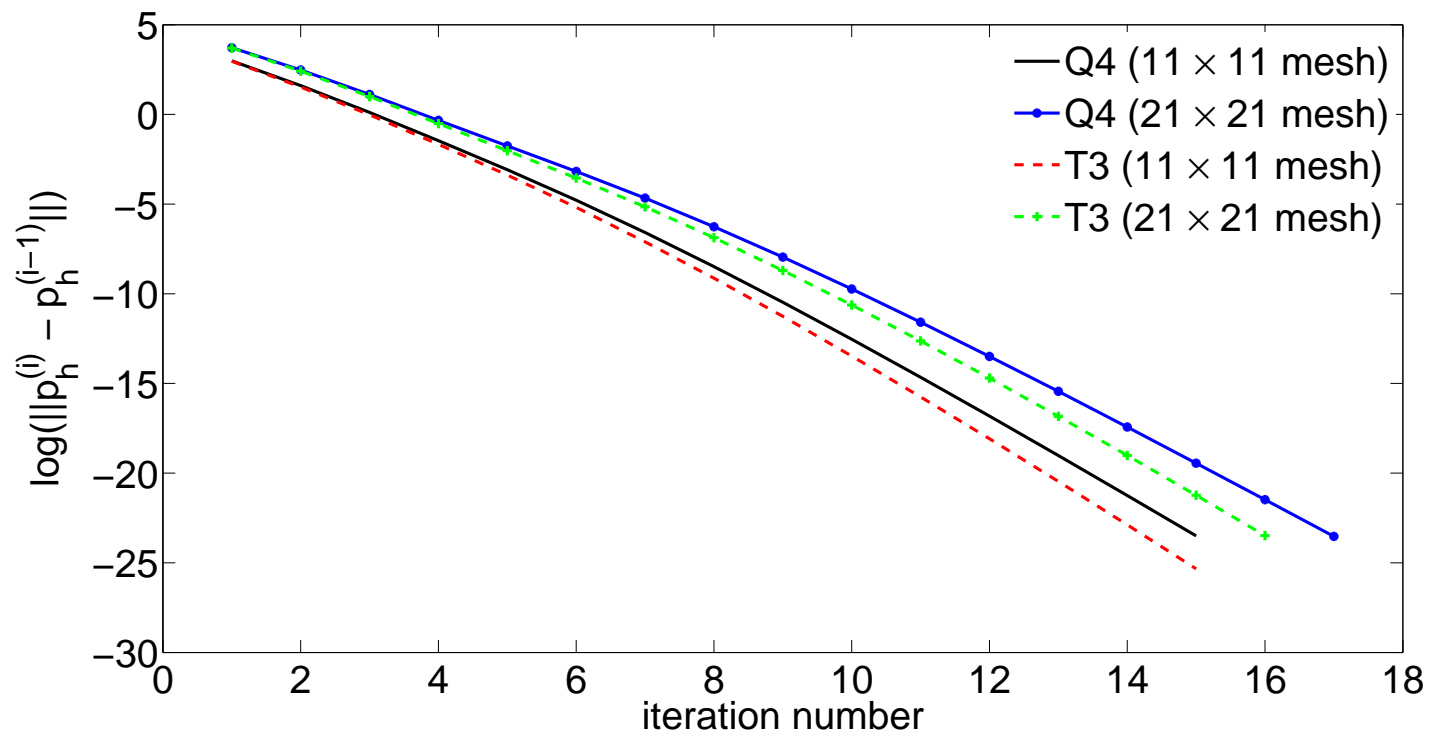

FiguRE 10. Five-spot problem: Variation of $\left\|p_{h}^{(i)}-p_{h}^{(i-1)}\right\|$ (which is based on the 2-norm of the nodal values of the pressure) with respect to iteration number using four-node quadrilateral (denoted by Q4) and three-node triangular (denoted by T3) elements. In this numerical simulation we have used $\epsilon_{\mathrm{TOL}}=10^{-10}$.

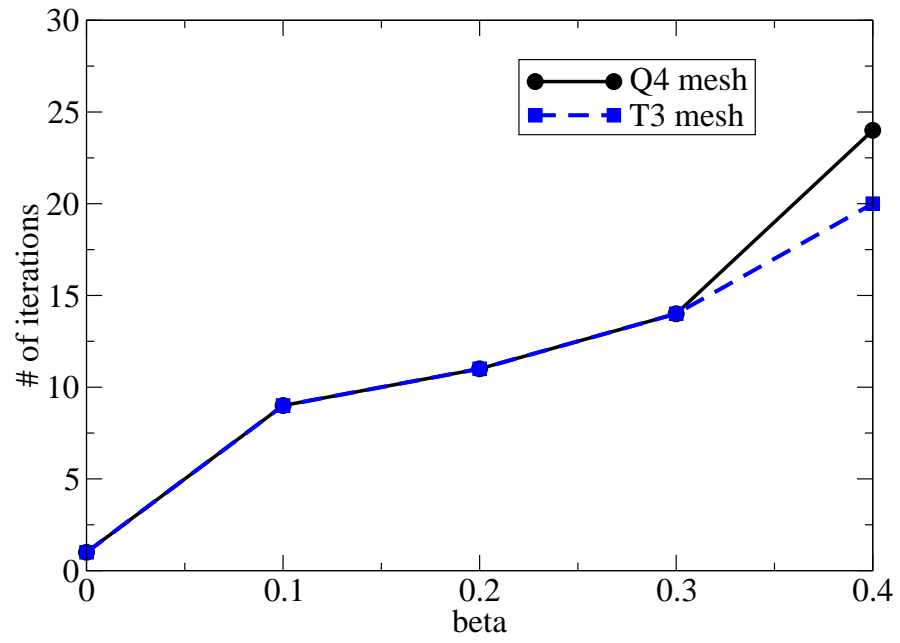

FiguRE 11. Five-spot problem: This figure shows the number of iterations taken by the numerical algorithm for various values of $\beta$, and for both Q4 and T3 meshes. Note that $\beta$ is an indicator of the strength of the nonlinearity. 


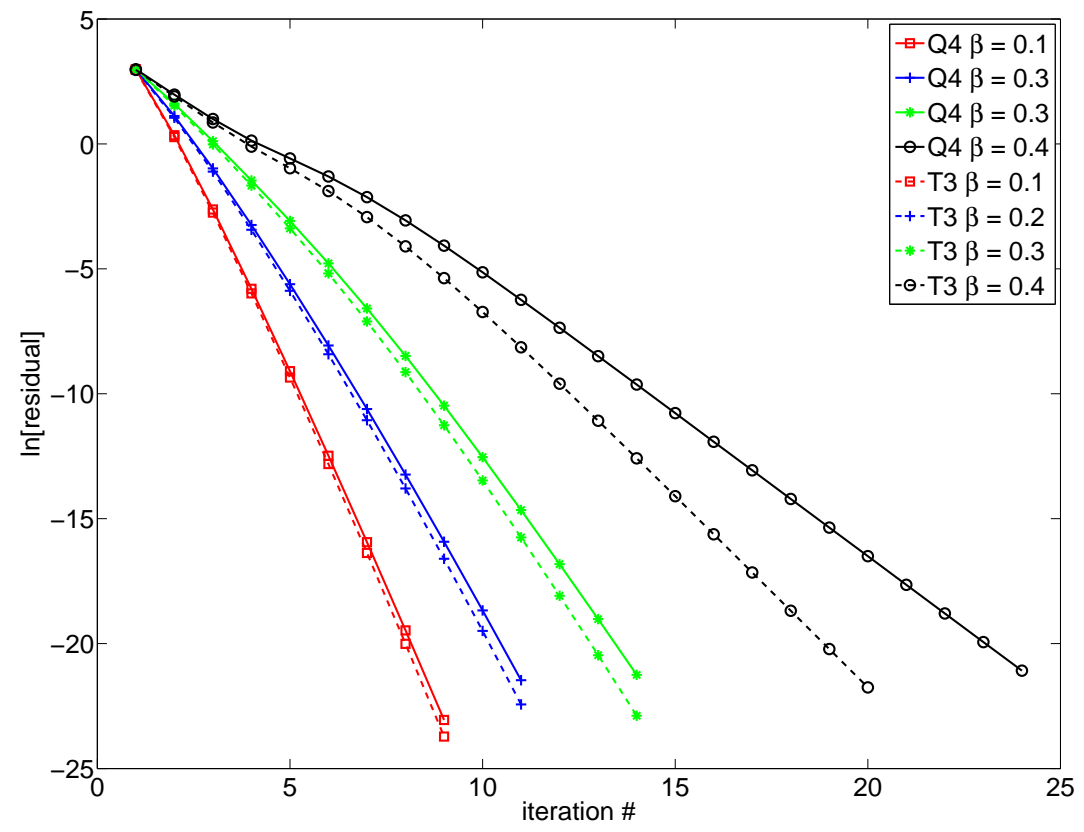

FiguRE 12. Five-spot problem: This figure shows the variation of the residual with respect to iteration number for various values of $\beta$, and for both Q4 and T3 meshes. Note that the $y$-axis is natural logarithm of the residual.

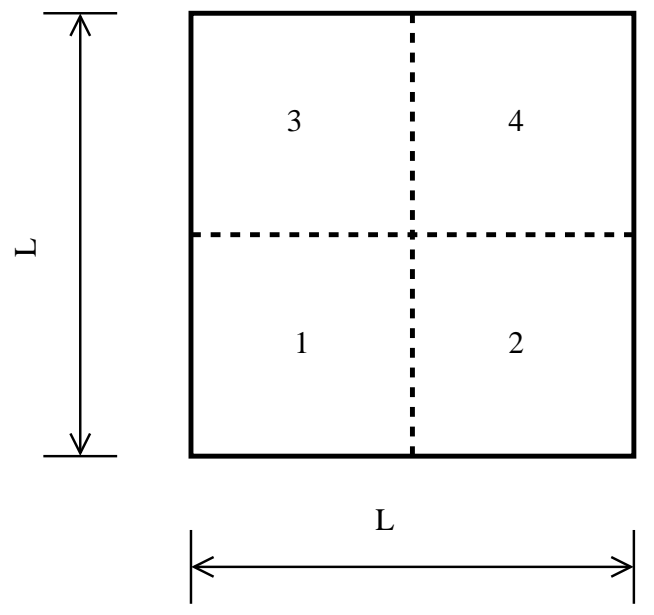

FiguRE 13. Checkerboard problem: A pictorial description. In regions I and IV, we have taken $\bar{\alpha}_{0}=1.0$; and in regions II and III, we have taken $\bar{\alpha}_{0}=0.001$. 


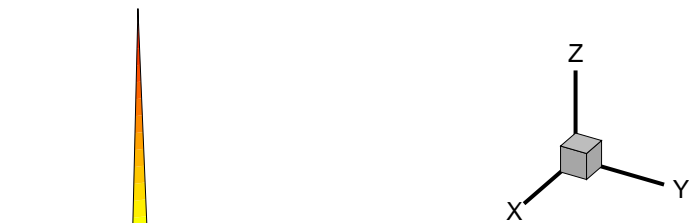

5.0

4.6

4.2

3.8

3.4

3.0

2.6

2.2

1.8

1.4

1.0
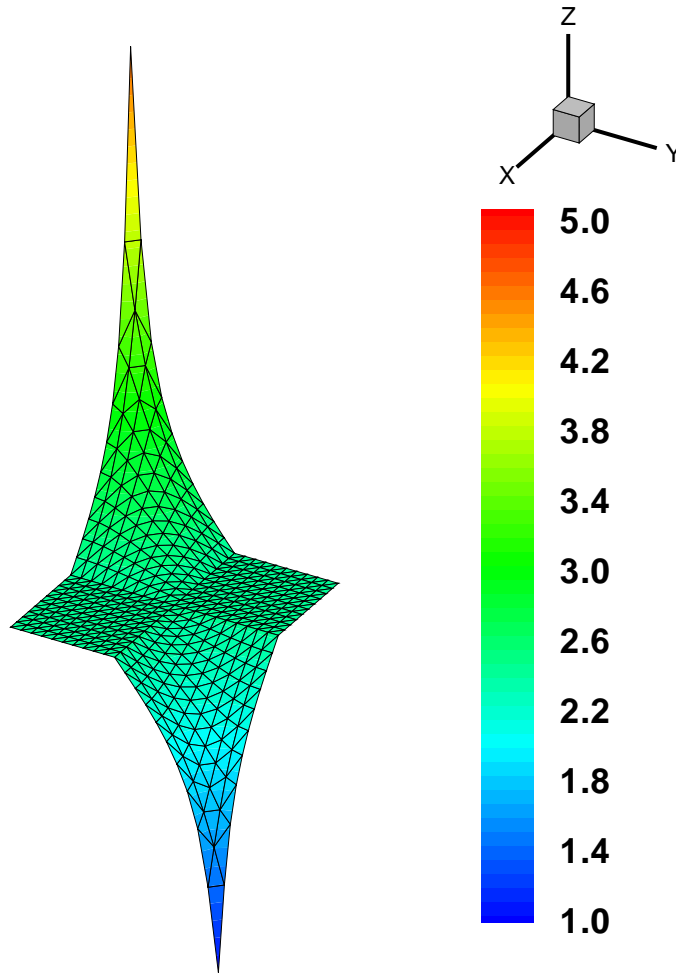

5.0

4.6

4.2

3.8

3.4

3.0

2.6

2.2

1.8

1.4

1.0

Figure 14. Checkerboard problem: Pressure contours using four-node quadrilateral (top) and three-node triangular (bottom) elements. We have used 21 nodes along each side of the computational domain. Barus formula with $\bar{\beta}=0.3, \mathcal{A}=1$ and $\mathcal{B}=0$ are employed. 


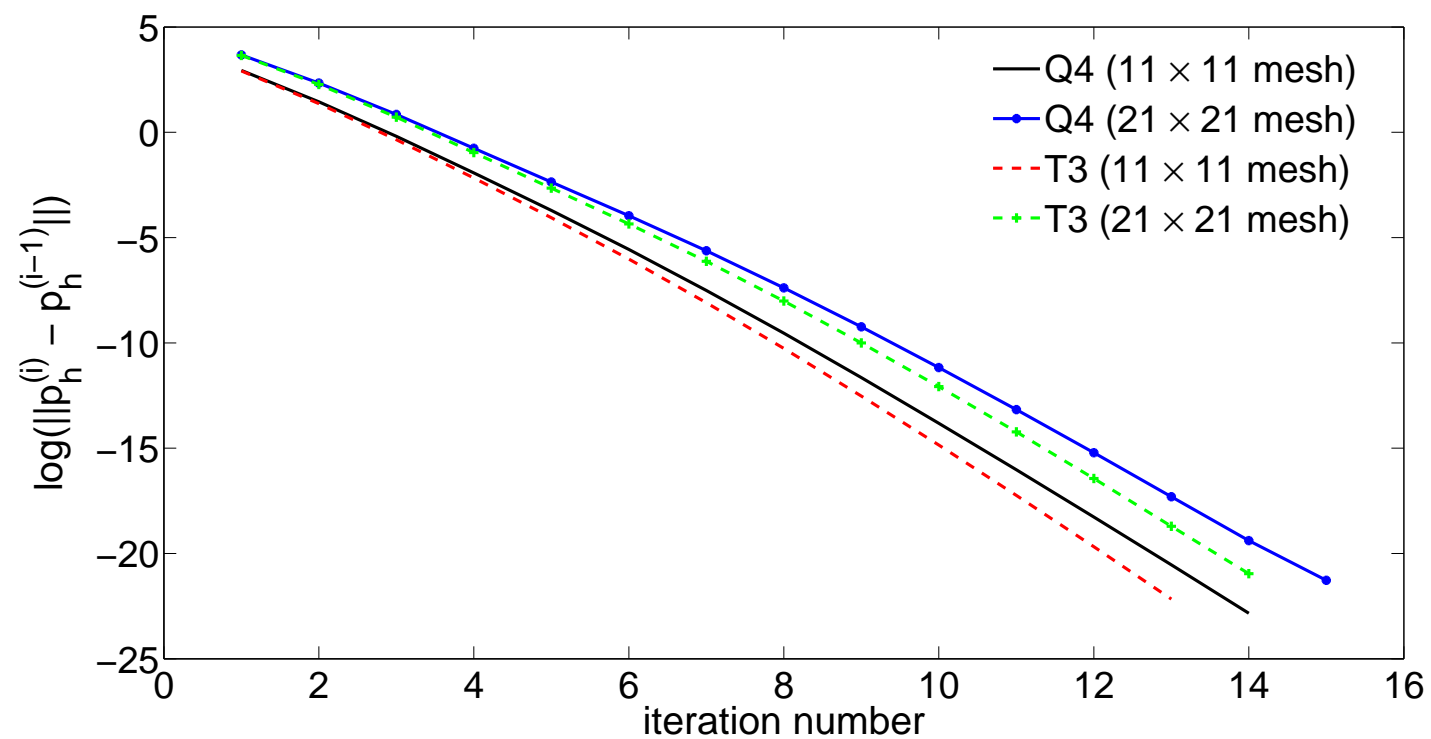

FIGURE 15. Checkerboard problem: Variation of $\left\|\bar{p}_{h}^{(i)}-\bar{p}_{h}^{(i-1)}\right\|$ (which is based on the 2-norm of the nodal values of the pressure) with respect to iteration number using four-node quadrilateral (denoted by Q4) and three-node triangular (denoted by T3) elements. In this numerical simulation we have used $\epsilon_{\mathrm{TOL}}=10^{-9}$, and $\bar{\beta}=0.3$.

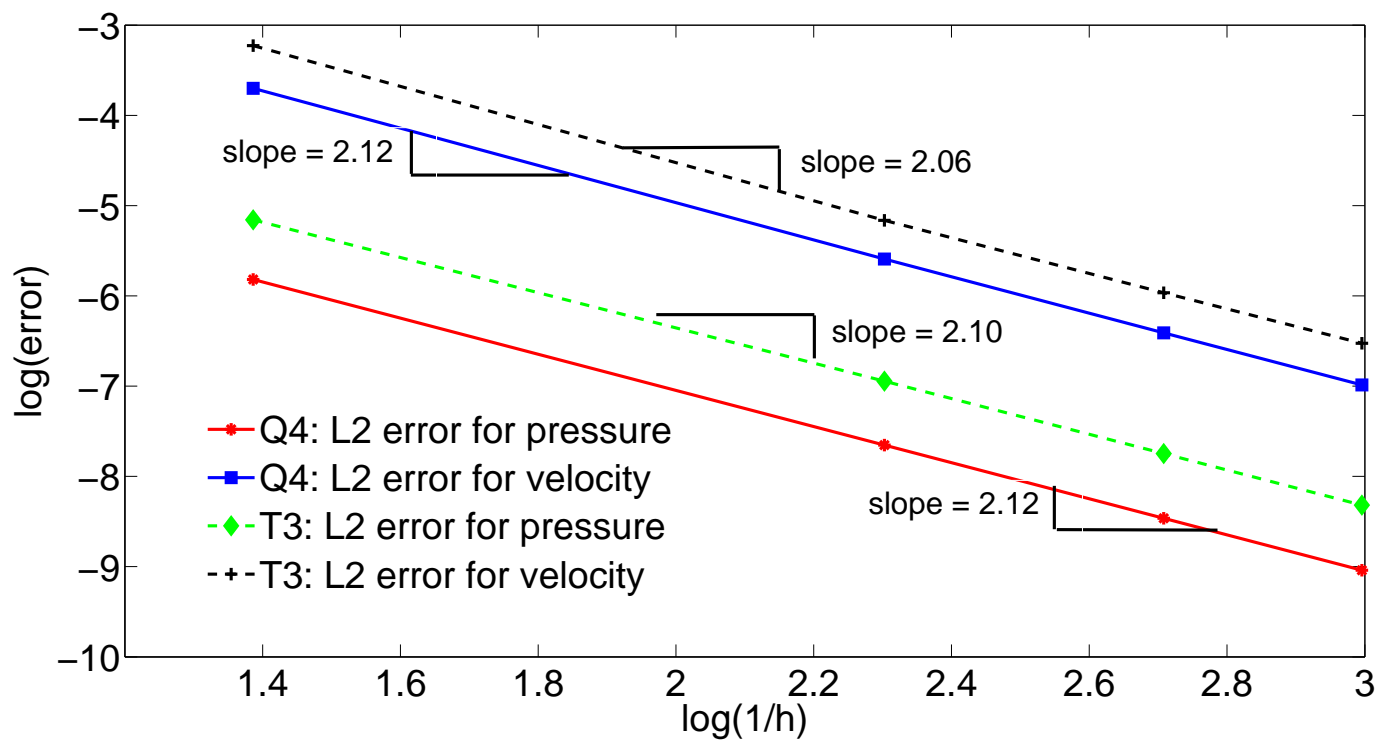

FIgURE 16. Numerical $h$-convergence studies: The figure shows the rate of convergence in $L_{2}$-norm for four-node quadrilateral (Q4) and three-node triangular (T3) elements. 


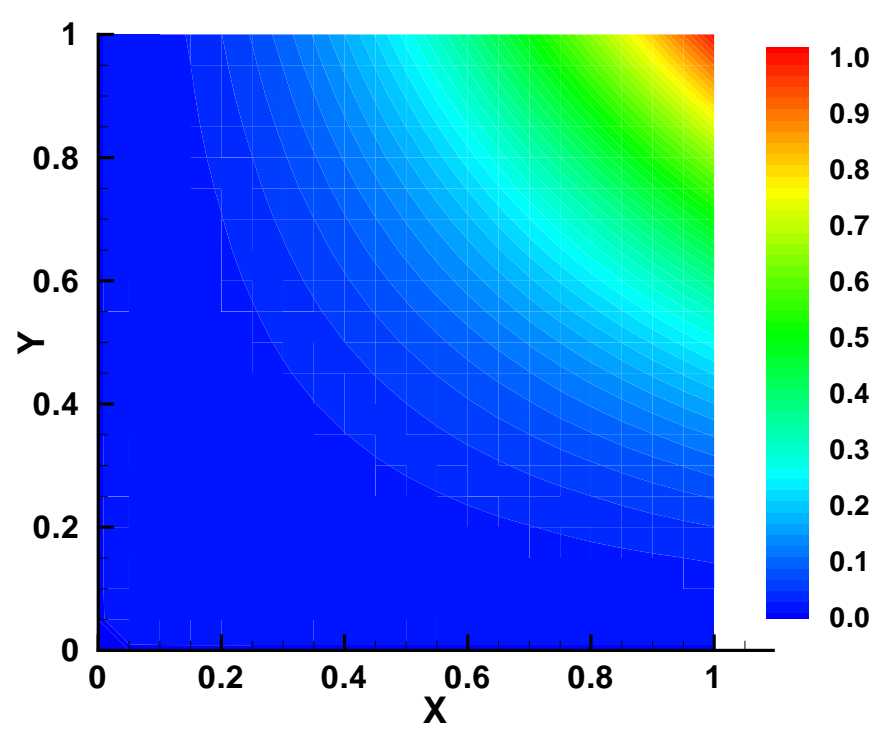

FiguRE 17. Numerical $h$-convergence studies: Pressure contours using $21 \times 21$ fournode quadrilateral mesh. We have employed equal-order interpolation for the velocity and pressure, and there are no spurious oscillations in the pressure. The analytical solution for pressure is given by $p(x, y)=x^{2} y^{2}$, and the numerical solution matched well with the analytical solution. 

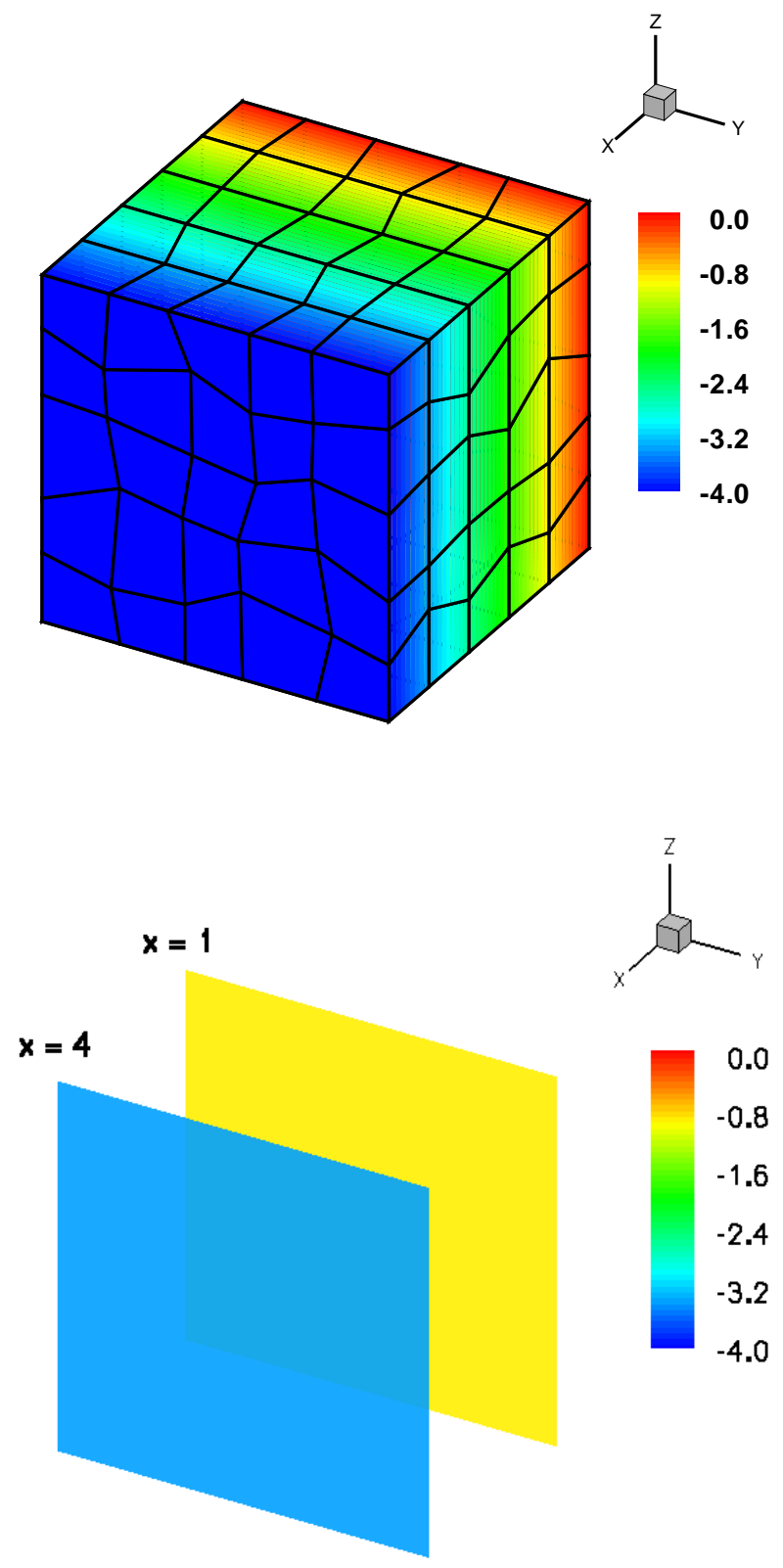

FiguRE 18. Three-dimensional constant flow: This figure shows the contours of pressure, and the mesh is also shown in the top figure. In the bottom figure we have shown the pressure on the $x=1$ and $x=4$ planes. In this numerical example we have employed Barus formula with $\alpha_{0}=1$ and $\beta=0.1$. 


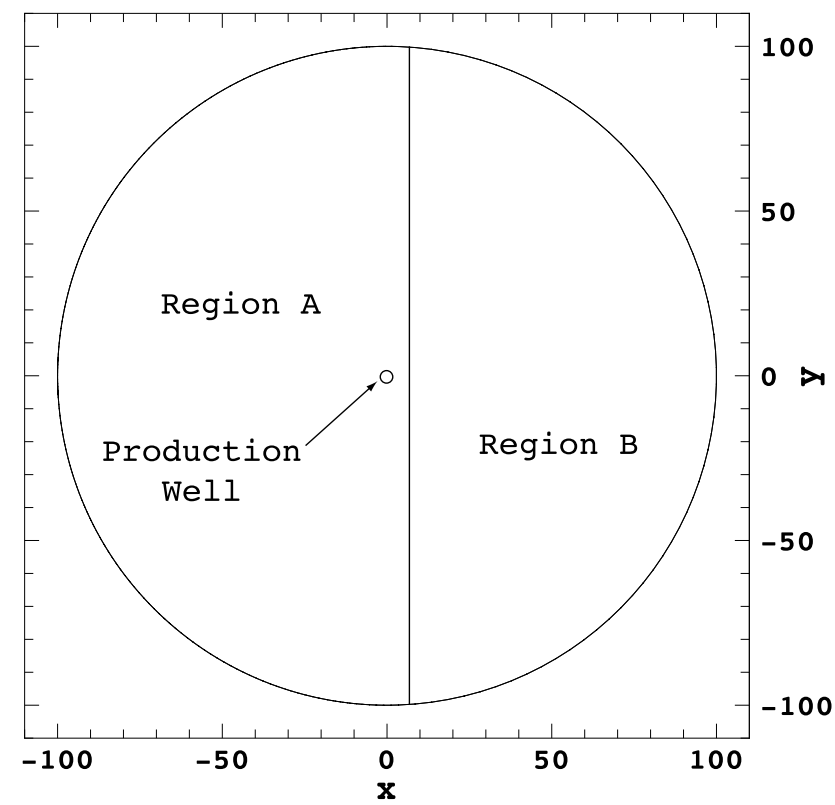

FiguRE 19. Regions with different permeability: This figure shows the computational domain and the location of production well.

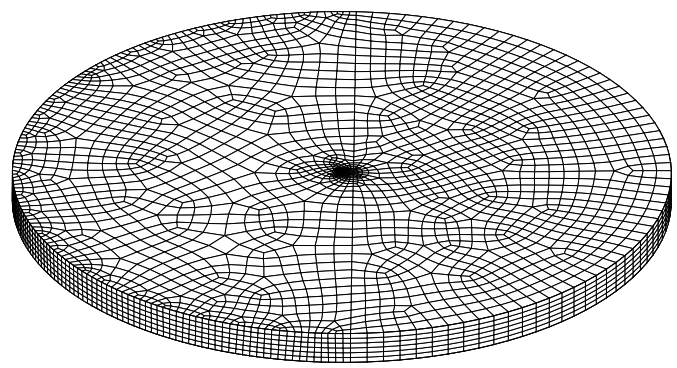

FiguRE 20. Regions with different permeability: Three-dimensional finite element mesh using eight-node (linear) brick elements.

Correspondence to: Dr. Kalyana Babu Nakshatrala, Department of Civil and Environmental Engineering, Engineering Building \#1, Room \#135, University of Houston, Houston, Texas - 77204. TEL: +1-713-743-4418

E-mail address: knakshatrala@uh.edu

Dr. Daniel Z. Turner, Department of Civil Engineering, University of Stollenbosch, StollenBosch, South AfricA. TEL: +27-21-808-4434

E-mail address: dzturner@sun.ac.za 


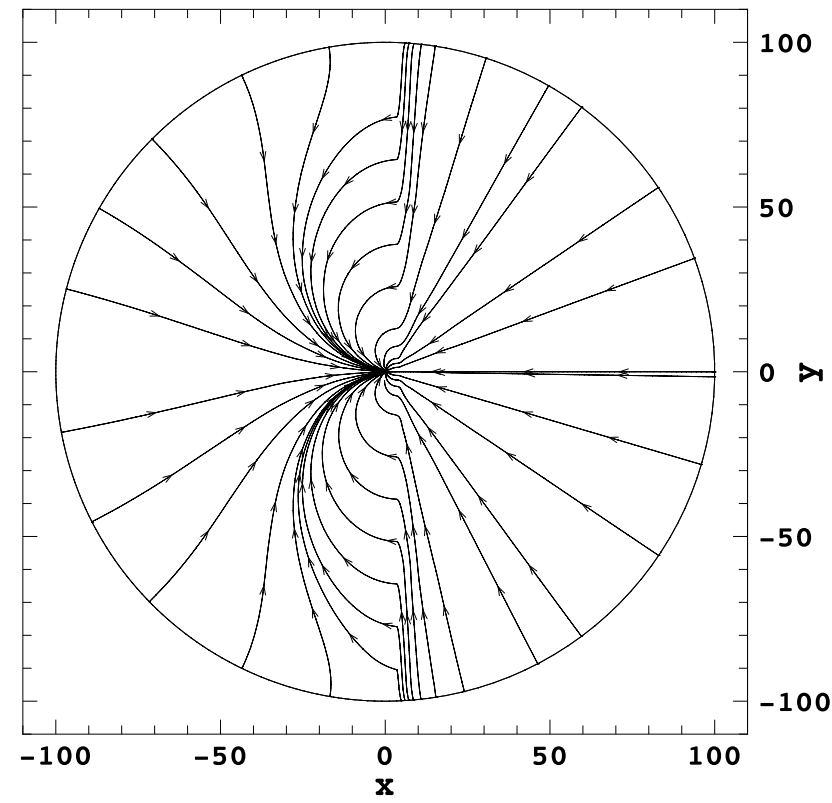

FIgURE 21. Regions with different permeability: This figure shows the velocity streamlines for $\beta=0$. (Only the streamlines for $\beta=0$ are plotted because the streamlines for all other values are similar.)

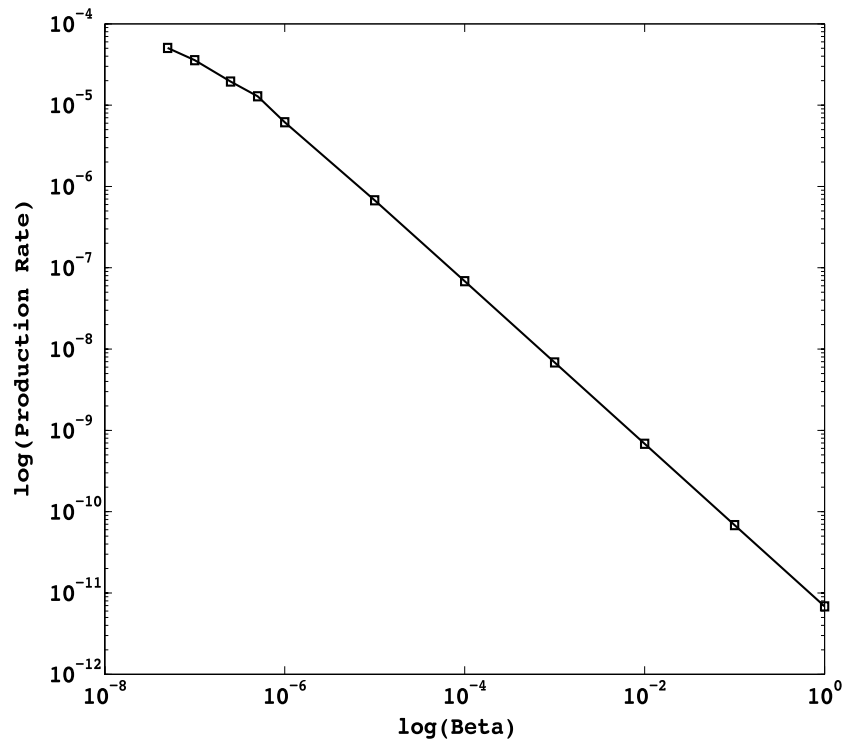

FiguRE 22. Regions with different permeability: This figure shows the production rate at the well opening for various values of $\beta$. 


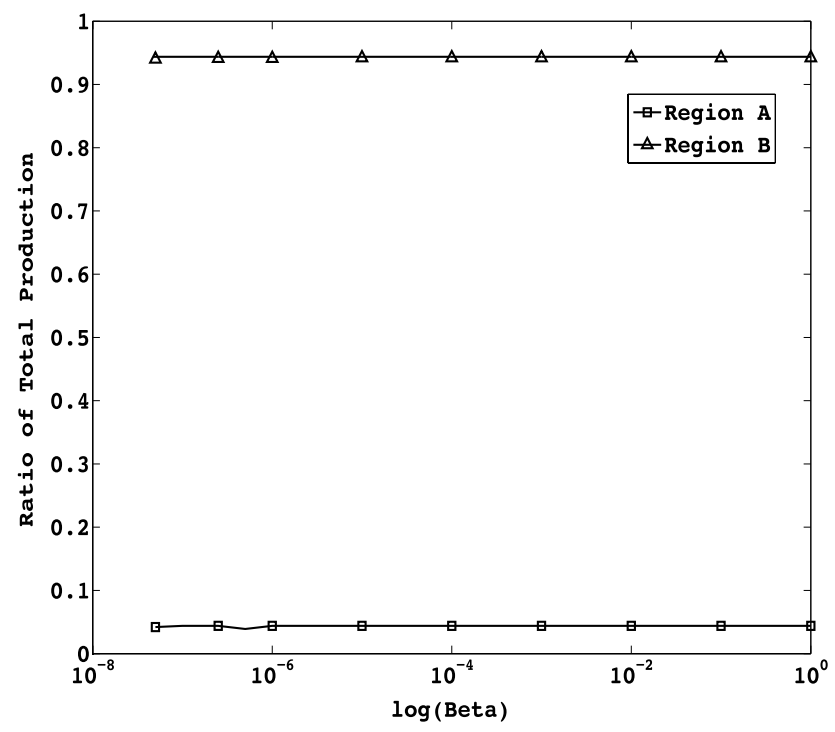

FiguRE 23. Regions with different permeability: This figure shows the ratio of the total production emanating from regions $\mathrm{A}$ and $\mathrm{B}$ with respect to $\beta$.

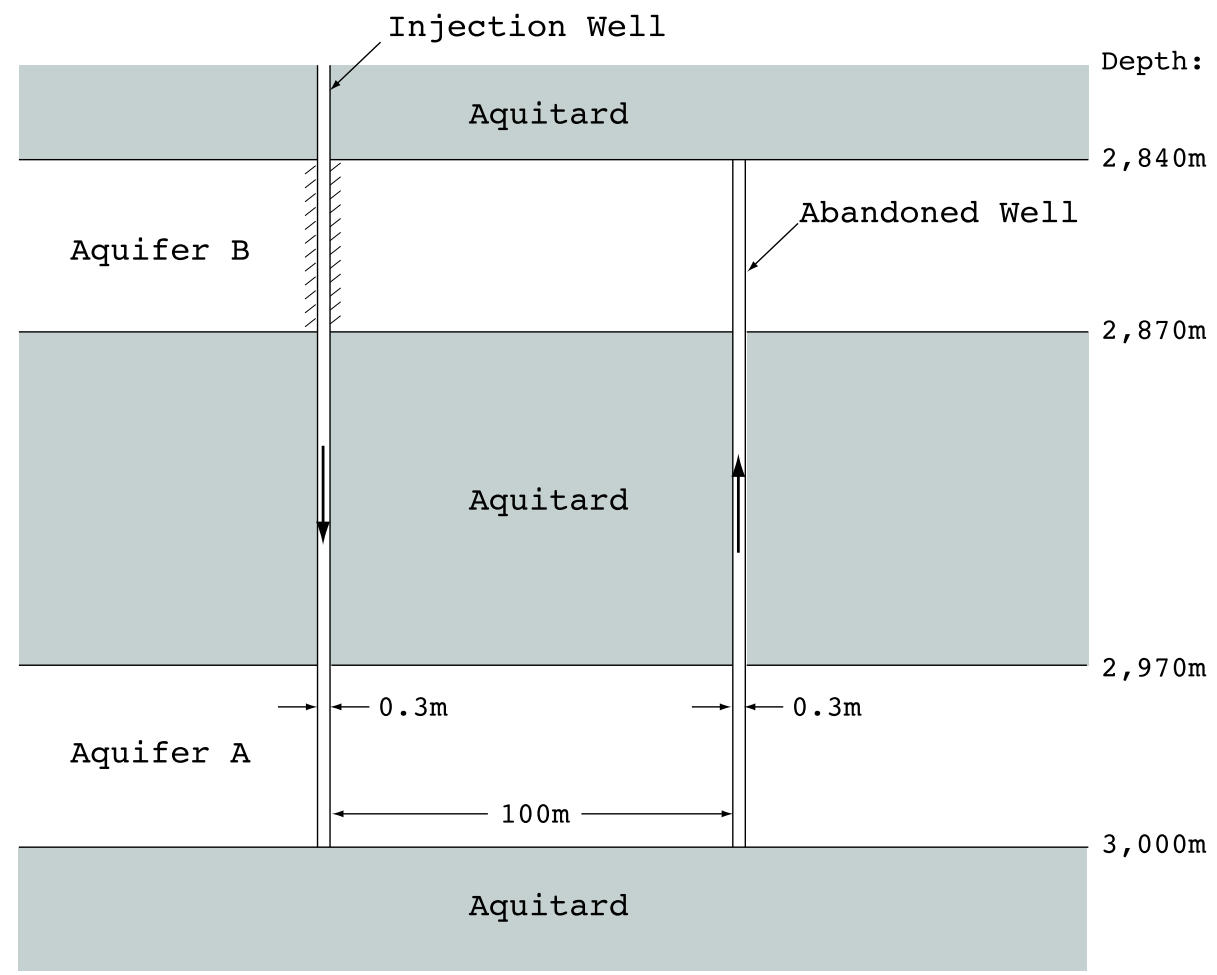

Figure 24. $\mathrm{CO}_{2}$ leakage through an abandoned well: A pictorial description of the problem showing the cross-sectional view along the plane containing the injection and abandoned wells. 

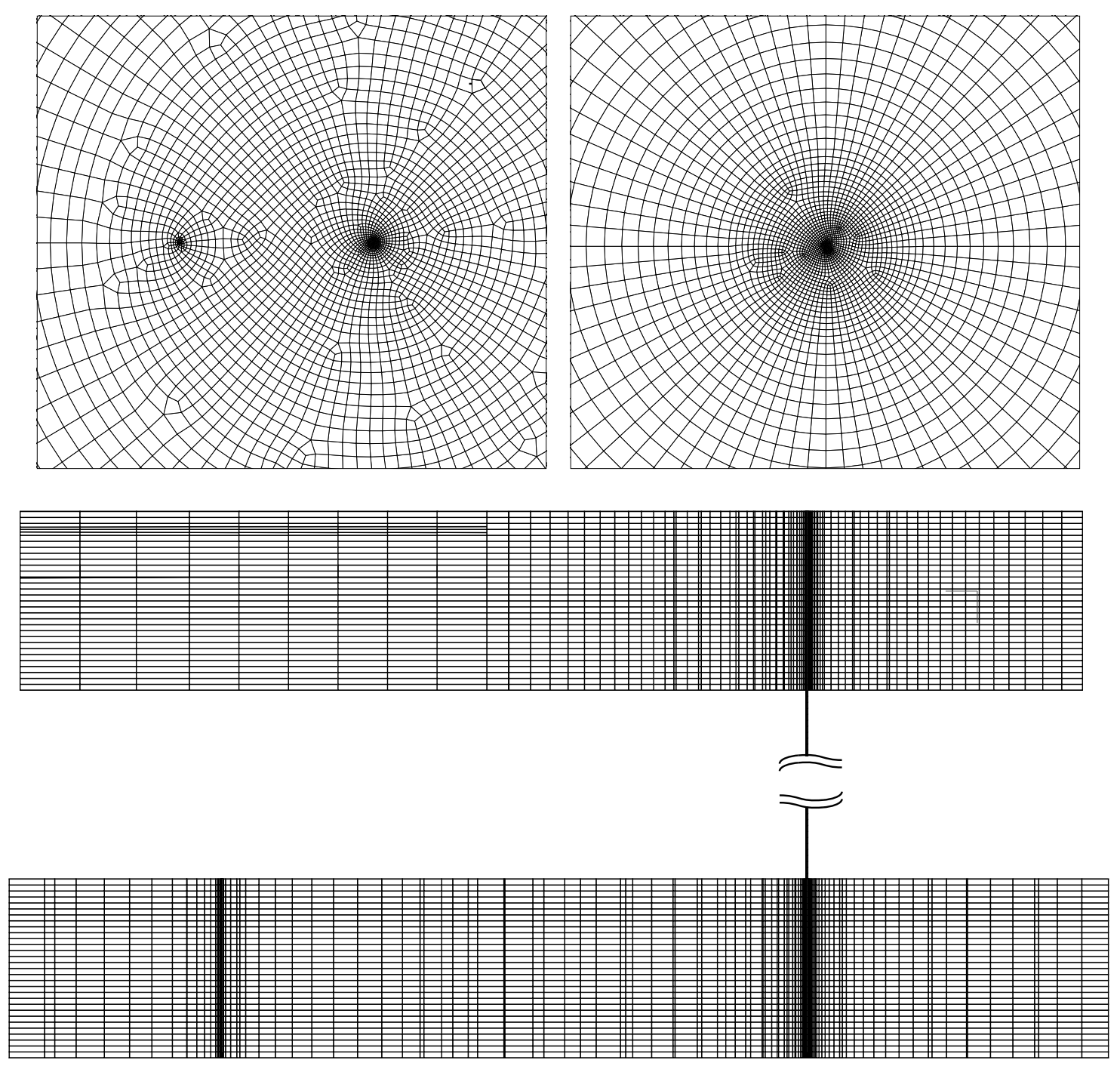

FiguRE 25. $\mathrm{CO}_{2}$ leakage through an abandoned well: This figure shows the computational mesh used in the numerical simulation. The top-left subfigure shows the mesh at the bottom of the domain, and the top-right subfigure shows the mesh at the top of the domain. The bottom subfigure shows the slice through the center near the injection and abandoned wells. There are over 1.14 million unknowns in this test problem. 

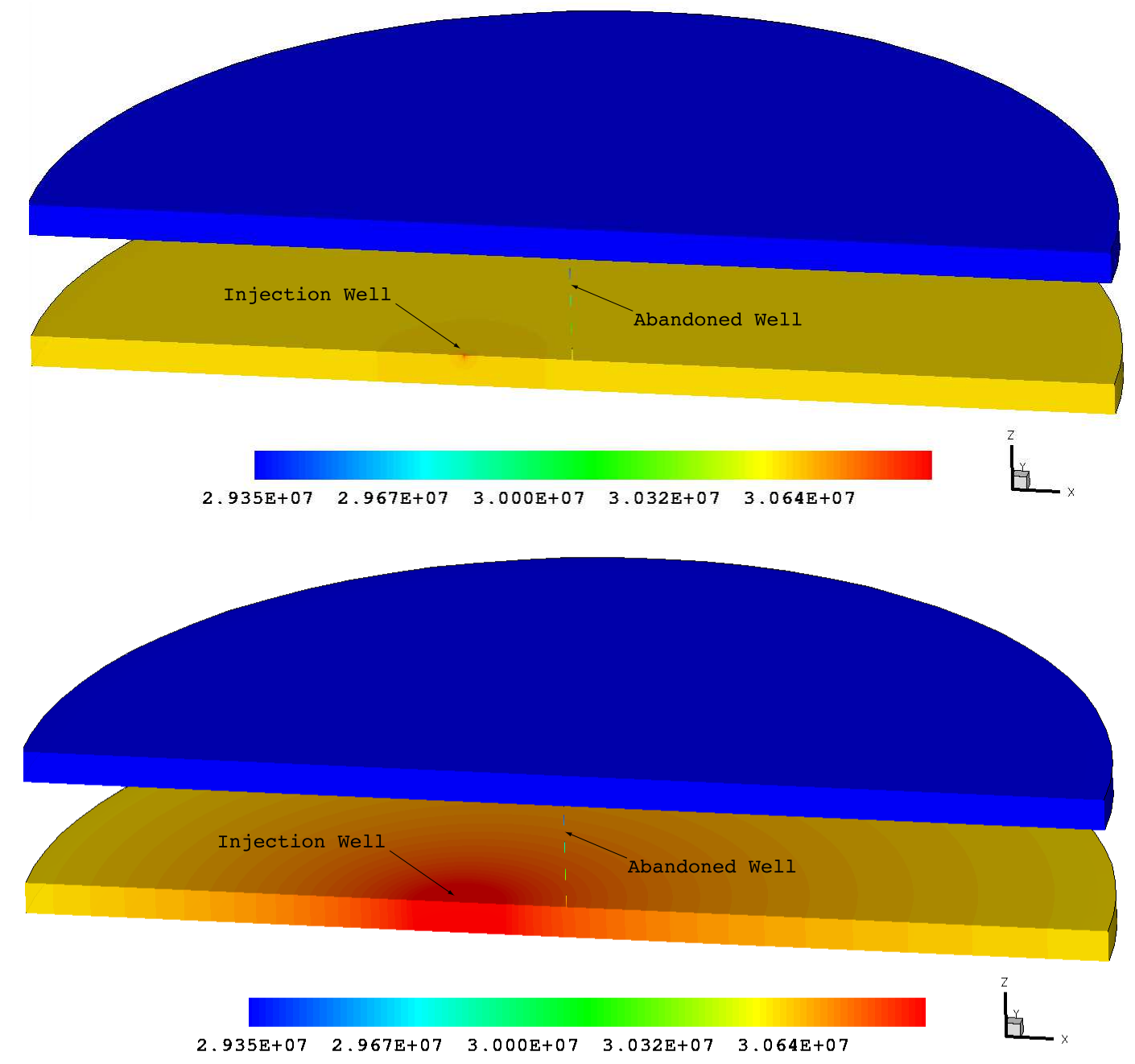

Figure 26. $\mathrm{CO}_{2}$ leakage through an abandoned well: This figure shows contours of the pressure for $\bar{\beta}=0$ (top) and $\bar{\beta}=1$ (bottom). It is evident from the figure that the modified Darcy model predicts higher pressures and higher pressure gradients than of Darcy model. Half of the domain has been removed to show the detail. 

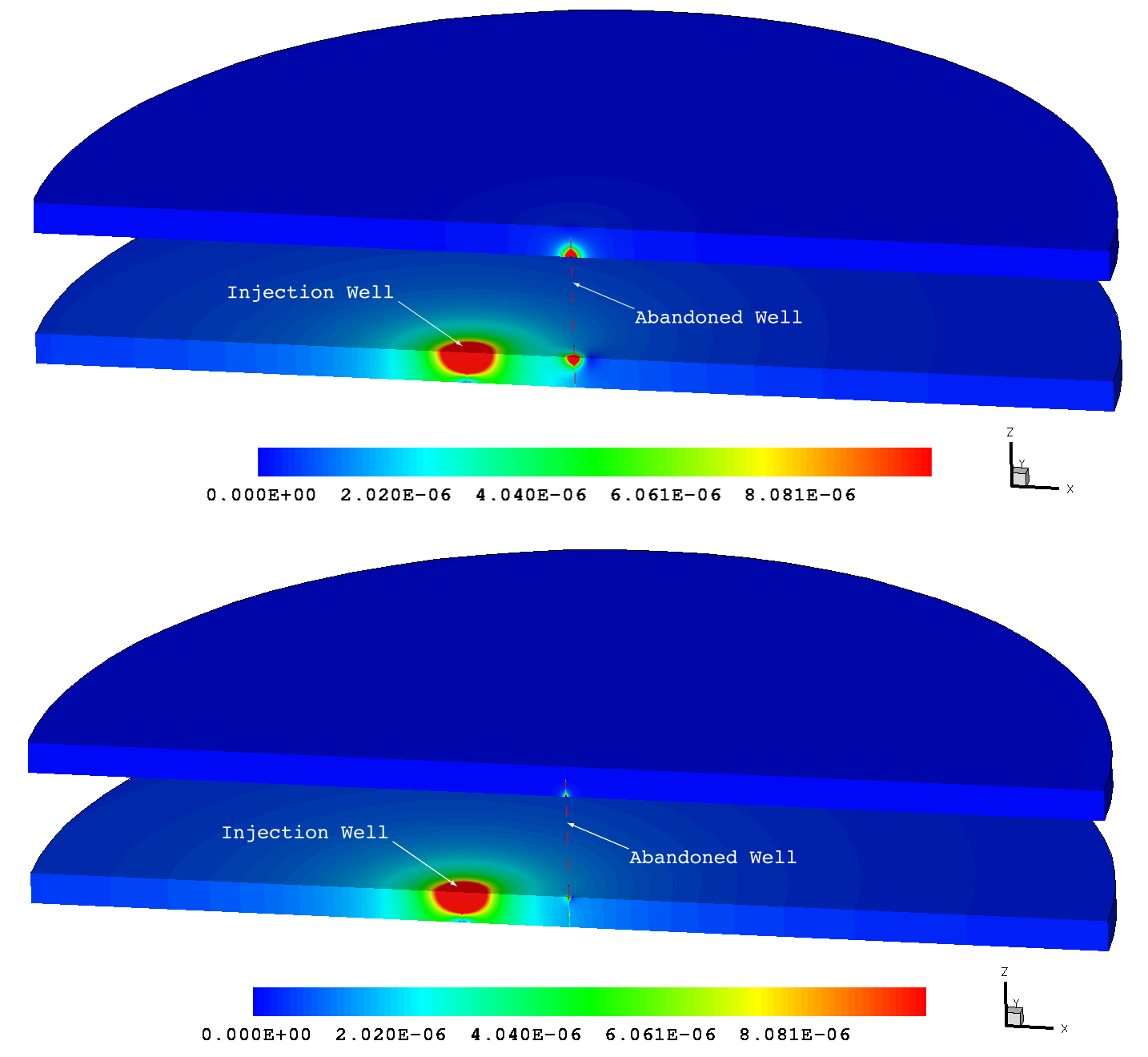

Figure 27. $\mathrm{CO}_{2}$ leakage through an abandoned well: This figure shows contours of the magnitude of the velocity for $\bar{\beta}=0$ (top) and $\bar{\beta}=1$ (bottom). It is evident from the figure that Darcy model predicts higher velocity in the abandoned well than the prediction made by the modified Darcy model. Half of the domain has been removed to show the detail. 


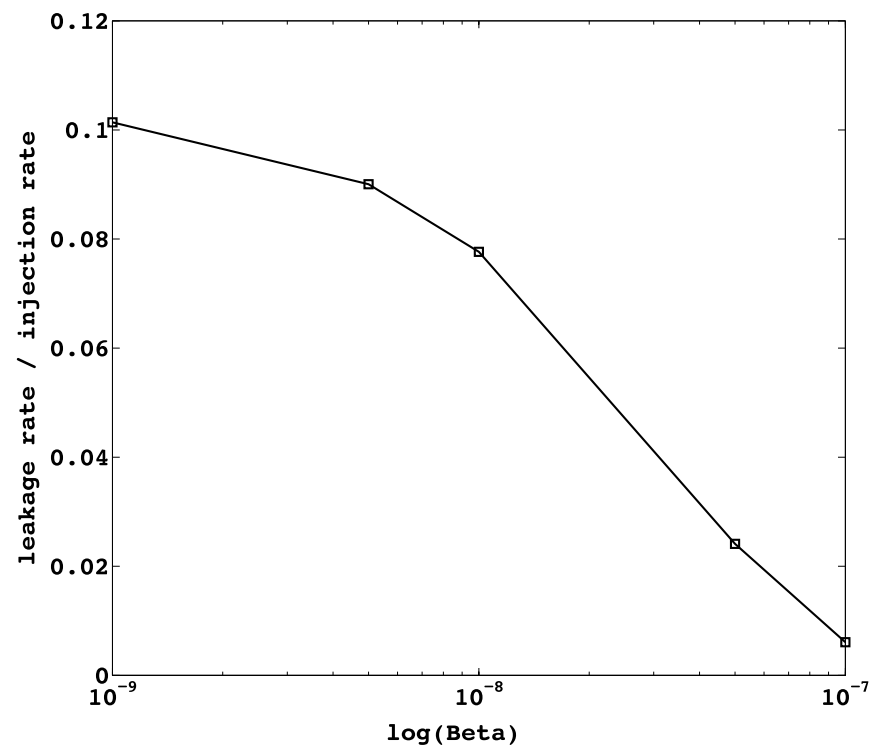

Figure 28. $\mathrm{CO}_{2}$ leakage through an abandoned well: This figure shows the ratio of leakage rate to injection rate versus $\beta$. Darcy model over-predicts the leakage rate than the modified Darcy model, and the ratio between the leakage rate and the injection rate decreases monotonically with increase in $\beta$. This discovery will be crucial in designing the seal in a geological carbon-dioxide sequestration geosystem. A design of the seal based on Darcy model will be too conservation, which may increase the expense of the geological carbon-dioxide sequestration project. 